Document downloaded from:

http://hdl.handle.net/10251/146291

This paper must be cited as:

Wrona, S.; Diego Antón, MD.; Pawelczyk, M. (11-2). Shaping zones of quiet in a large enclosure generated by an active noise control system. Control Engineering Practice. 80:116. https://doi.org/10.1016/j.conengprac.2018.08.004

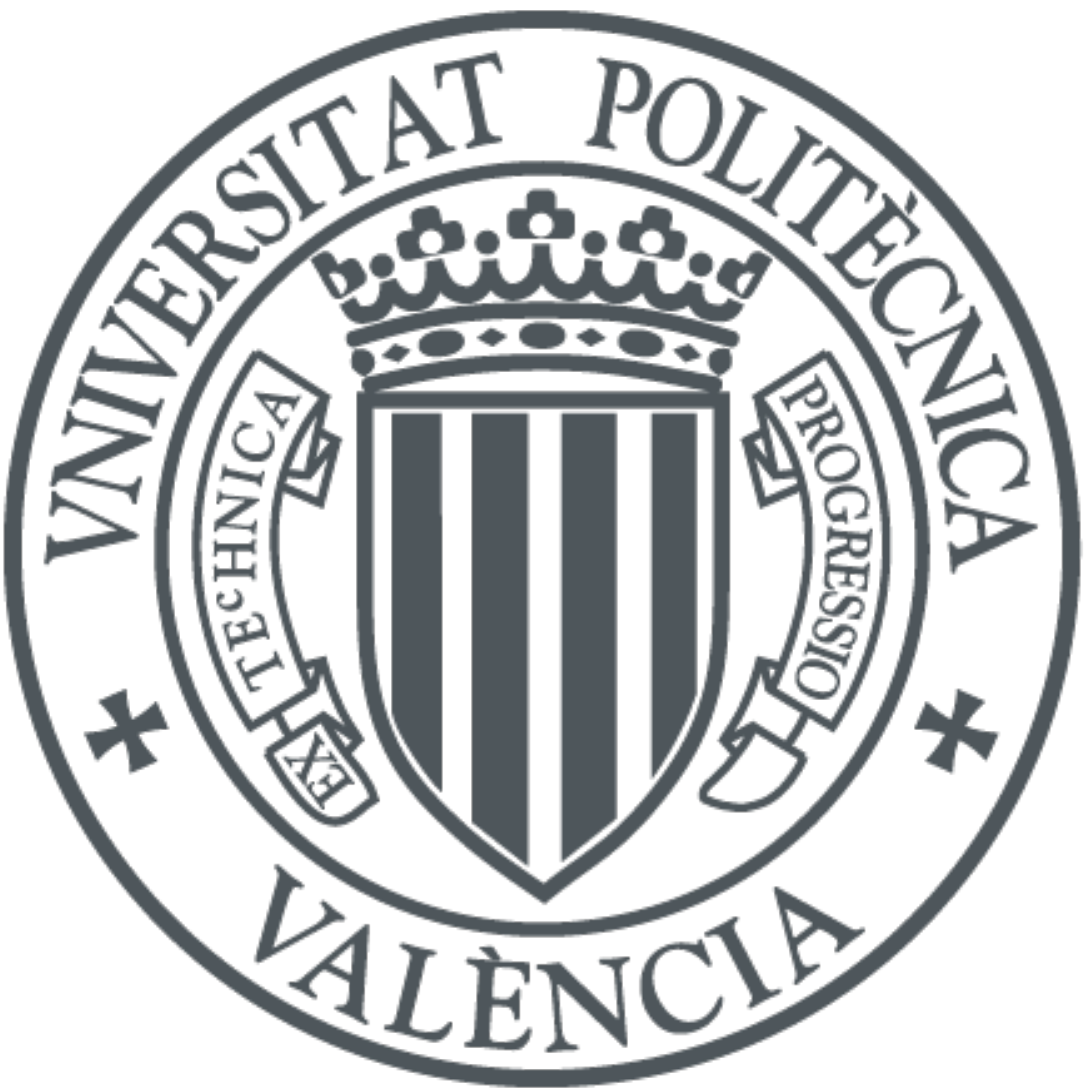

The final publication is available at

https://doi.org/10.1016/j.conengprac.2018.08.004

Copyright Elsevier

Additional Information 


\title{
Shaping zones of quiet generated by an active noise control system
}

\author{
Stanislaw Wrona ${ }^{\mathrm{a}}$, Maria de Diego ${ }^{\mathrm{b}}$, Marek Pawelczyk ${ }^{\mathrm{a}}$ \\ ${ }^{a}$ Institute of Automatic Control, Silesian University of Technology, ul. Akademicka 16, 44-100 Gliwice, Poland \\ ${ }^{b}$ Institute of Telecommunications and Multimedia Applications, Universitat Politecnica de Valencia, \\ Camino de Vera s/n; 46022 Valencia, Spain
}

\begin{abstract}
Performance of an Active Noise Control (ANC) system strongly depends on sensors and actuators spatial arrangement. It determines both achieved Noise Reduction (NR) levels and spatial distribution of obtained zones of quiet, making it an essential problem. However, if the acoustic field in the enclosure can be appropriately modelled, then optimization algorithms can be employed to find efficient configuration of the ANC system, enhancing its performance according to a formulated cost function and constraints. This paper proposes a complete method for enhancing NR levels and shaping zones of quiet generated with an ANC system by optimization of sensors and actuators arrangement. A Memetic Algorithm (MA) is utilized. The MA itself and its proposed operators are described. The optimization problem formulation is derived and discussed. As a control algorithm, Distributed Multiple Error Filtered-x Least Mean Square (DMEFxLMS) is used. Extensive simulation results are presented for an exemplary real enclosure. The model of the acoustic environment has been obtained by real-world experiments, resulting in identification of 36864 acoustic responses in total. Practically feasible cost function and constraints are evaluated. Advantages and limits of the method are pointed out and discussed.
\end{abstract}

Keywords: Acoustic control, Adaptive filters, Optimization process, Sensors and actuators arrangement, Noise reduction

Email addresses: stanislaw.wrona@polsl.pl (Stanislaw Wrona), mdediego@dcom.upv.es (Maria de Diego), marek.pawelczyk@polsl.pl (Marek Pawelczyk) 


\section{Introduction}

An excessive acoustic noise is an important problem in the modern society and it stimulates the development of a variety of noise reduction techniques. A common solution is to apply passive methods, such as the use of sound-absorbing/sound-insulating materials. They constitute a satisfactory solution to many noise problems. However, they are ineffective for noise at low frequencies. Moreover, they often are inapplicable due to unacceptable increase in mass and volume, and introduction of an additional heat insulation. An alternative approach is to use active control methods, by applying a set of sensors and actuators, and running a control algorithm [1, 2, 3, 4]. Active methods are better suited for reduction of low frequency noise [5], and do not introduce heat transfer problems.

One of the active control approaches is the Active Noise Control (ANC). The ANC methods can be explained by the principle of superposition, where in contrast to passive methods, additional energy is introduced to the system through a set of control inputs (secondary sources) to obtain a secondary response that adds to the primary disturbance field. In result, the total response of the system can be reduced (destructive interference) or altered in the desired manner. Typically, ANC systems consist of (i) an electronic controller and (ii) a set of sensors and actuators, usually microphones and loudspeakers. The controller drives actuators basing on sensors signals. It often runs in an adaptive way to follow changes in the primary disturbance field or secondary transfer path.

Performance of an ANC system strongly depends on sensors and actuators spatial arrangement in an acoustic environment. It determines both achieved Noise Reduction (NR) levels and spatial distribution of obtained zones of quiet, making it an essential problem. Moreover, there are often multiple constraints imposed on admissible sensors and actuators configuration, making the problem even more complex and difficult to solve. However, if the acoustic environment can be modelled (by theoretical calculations or experimental measurements), then optimization algorithms can be employed to find efficient configuration of the ANC system, enhancing its performance according to a formulated cost function.

Optimization techniques have been used over the years to enhance performance of ANC systems. The use of a genetic algorithm to optimize loudspeakers locations of an ANC system in a telephone kiosk is reported in [6]. The authors utilized the average of acoustical potential energy reduction as a performance index. The optimization of arrangement of ANC system secondary 
sources around a single monopole primary source is shown in [7]. The authors considered minimization of radiated sound power in the free field. The optimization of microphones and speakers arrangement of an ANC system in an aircraft is presented in [8]. In the employed genetic algorithm, the average noise reduction obtained by the ANC was used as a fitness function. Secondary sources and error sensors locations are optimized in [9], considering the summation of the squared pressure at all evaluated points and the total control energy in the cost function. Other reports of ANC system configuration optimization are given e.g. in [10, 11, 12]. The most of the cited reports consider optimization with indices describing the ANC system global performance (in the whole room, enclosure or space). However, in many applications such global reduction is unfeasible and unnecessary. Hence, it is practically sensible to define the target zones where the noise reduction is truly required (where people are often present) and neglect the control results elsewhere (where people are rarely or only for short periods of time). In general, such approach results in the lesser necessary number of sensors and actuators, thus reducing control unit computational burden and energy effort. But in order to satisfy the application-related requirements of noise reduction, the generated zones of quiet should be appropriately shaped to fit the target zones. If necessary, the zones can follow people moving in the enclosure, based on different kind of sensors localizing them.

This paper proposes a complete, ready to apply, method for enhancing NR levels and shaping spatial distribution of zones of quiet generated with an ANC system by optimization of sensors and actuators arrangement. The method is based on an optimization approach utilizing a Memetic Algorithm (MA). The MA itself and its proposed operators are described in details. The proposed method employs a model of the acoustic environment and evaluates the ANC system in various configurations, according to the optimization procedure. As a result, an efficient arrangement is found that optimizes the specified cost function. The major differences of this work comparing with the previous ones are: (i) the precisely designed spatial distribution of target zones is embedded in the cost function (leading to shaped zones of quiet), instead of employing a scalar global performance index; (ii) the computationally-efficient Memetic Algorithm is used to find optimal solution, which operators are proposed and discussed in details. The optimization problem formulation is derived and discussed.

To facilitate presentation of the proposed method, an exemplary scenario is introduced. The scenario considers a room with a device generating excessive noise. In the room a user of the 
device is present and the goal is to generate a zone of quiet around listener's location (reducing the noise that he is exposed to). The scenario is used to investigate the proposed shaping method performance by simulation studies. A model of the acoustic environment is obtained by experimental measurements (a real room located at the Audio Processing Laboratory of the Polytechnic University of Valencia has been utilized for this purpose, which was equipped with 96 loudspeakers generating acoustic excitation and 384 microphones locations acquiring the responses, what resulted in identification of 36864 acoustic responses in total, available at www.gtac.upv.es/room.php). Multiple scenario variants, parameters and imposed constraints are tested. As a control algorithm, Distributed Multiple Error Filtered-x Least Mean Square (DMEFxLMS) is employed [13, 14].

This paper is organized as follows. Subsection 2.1 introduces the exemplary scenario, which constitutes a basis for explanation of the proposed method. Subsection 2.2 is devoted to the ANC system that is utilized in the presented research. Then, Subsection 2.3 formulates the problem of sensors and actuators arrangement optimization. Section 3 is devoted to the shaping method itself. Firstly, an overview of the proposed method is given. Subsequently, the modelling of the acoustic environment is discussed. Then, several feasible cost functions are proposed. Afterwards, the employed memetic algorithm is described, along with its proposed operators. Then, Section 4 presents assumptions adopted for simulations presented in this paper. The parameters of the ANC system and the shaping method are given. Then, in Section 5 the obtained simulation results are presented. Temporal, spatial and frequency characteristics are shown. An impact of three important parameters on the obtained noise reduction results is studied. Finally, advantages and limits of the proposed approach are pointed out and discussed, and conclusions for future research are drawn.

\section{Problem description}

\subsection{The exemplary scenario}

For the sake of clarity, the proposed method is presented in this paper on the basis of an exemplary scenario, although, the presented considerations preserve generality. In the example, a large room with a disturbance source - a device generating an excessive broadband noise, is considered. It is a situation commonly encountered in real life. The aim is to reduce the noise for which the user of the device is exposed to (they have to be present in the room with the 
device). It is assumed that passive methods are insufficient in this case (low frequencies are dominating in the noise spectrum, and the users cannot be separated from the noisy device by a barrier), therefore the ANC system is introduced. Its goal is to generate a zone of quiet around a known location (potential user location) with a limited number of sensors, actuators, and their admissible locations. The objective of the optimization process is to find efficient arrangement of sensors and actuators, enabling the control algorithm to achieve the highest possible NR levels within the target zone, while outside the zone noise enhancement is allowed. It is a practically feasible approach, as the global noise reduction with ANC system in real applications represents very high energy demand and is very difficult (or even impossible) to obtain due to technological limitations [5]. On the other hand, global noise reduction is often not needed. Hence, a distribution of target zones should be defined, where the noise should be reduced, neglecting the effects obtained in other areas (where the presence of people is unlikely). The proposed shaping method is employed to achieve the goal.

An alternative active control approach worth mentioning, is the employment of an active casing, which relies on actively controlling vibration of the device casing with bonded actuators so that the emitted noise to the environment is reduced. Under certain conditions, it can provide global noise reduction with low energy demand. However, this approach is out of the scope of this paper and the reader is referred to other publications dedicated to it [15, 16, 17, 18].

\subsection{The ANC system}

\subsubsection{Control algorithm}

The control algorithm implemented in the ANC system controller is the DMEFxLMS [13]. It is based on the Multiple Error Filtered-x Least Mean Square (MEFxLMS) that has been proven to perform very well for ANC systems with multiple sensors and actuators [14]. The DMEFxLMS has been implemented with a collaborative incremental strategy in the network. The ANC system consists of $N_{n}$ separate nodes communicating and collaborating with each other to achieve a common goal. Each node consists of a controller and its own sensors and actuators. However, no communication constraints have been imposed in the network in this research, hence, the control system exhibits the same performance as the centralized implementation. In this research, it is assumed that the network is homogeneous and each node consists of one sensor and one actuator. Schematic representation of the control system of an individual node is given in Fig. 1 Fundamental issues of this algorithm are recalled below to set a reference for further reading. 


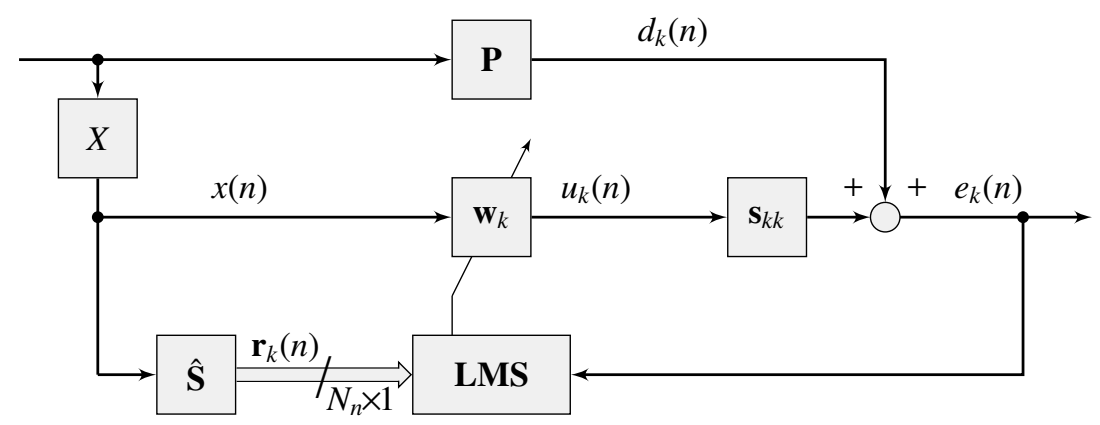

Figure 1: Control system of an individual $k$ th node.

For a detailed description of the DMEFxLMS algorithm that can handle different numbers of microphones and loudspeakers within a node, the reader is referred to [13].

The control filters in the whole network are updated at the $n$th sample according to the formula:

$$
\mathbf{w}(n)=\alpha \mathbf{w}(n-1)-\mu \sum_{k=1}^{N_{n}} \mathbf{r}_{k}(n) e_{k}(n)
$$

In Eq. 11, $\mathbf{w}(n)=\left[\mathbf{w}_{1}^{T}(n), \mathbf{w}_{2}^{T}(n), \ldots, \mathbf{w}_{N_{n}}^{T}(n)\right]^{T}$ is the global control filter vector, containing $N_{n}$ concatenated control filters, where $k$ th filter $\mathbf{w}_{k}(n)$ is used at $k$ th node. The control filter $\mathbf{w}_{k}(n)=\left[w_{k, 1}(n), w_{k, 2}(n), \ldots, w_{k, L_{w}}(n)\right]^{T}$ is the adaptive Finite Impulse Response (FIR) filter at sample $n$, and $L_{w}$ is the filter order. $0 \ll \alpha<1$ is the leakage coefficient and $\mu$ is the stepsize. $\mathbf{r}_{k}(n)=\left[\mathbf{r}_{1 k}^{T}(n), \mathbf{r}_{2 k}^{T}(n), \ldots, \mathbf{r}_{N_{n} k}^{T}(n)\right]^{T}$ is constructed similarly to $\mathbf{w}(n)$, where vector $\mathbf{r}_{i k}(n)=$ $\left[r_{i k}(n), r_{i k}(n-1), \ldots, r_{i k}\left(n-\left(L_{w}-1\right)\right)\right]^{T}$ is a vector of regressors of the $i k$ th filtered-reference signal. $e_{k}(n)$ is the $k$ th error signal obtained at the $k$ th node.

The $i k$ th filtered-reference signal is calculated as:

$$
r_{i k}(n)=\hat{\mathbf{s}}_{i k}^{T} \mathbf{x}_{r}(n),
$$

where $\hat{\mathbf{s}}_{i k}=\left[\hat{s}_{i k, 1}, \hat{s}_{i k, 2}, \ldots, \hat{s}_{i k, L_{s}}\right]^{T}$ is the vector of coefficients of the $L_{s}$ th order FIR model of the $i k$ th secondary path; and $\mathbf{x}_{r}(n)=\left[x(n), x(n-1), \ldots, x\left(n-\left(L_{s}-1\right)\right)\right]^{T}$ is a vector of regressors of the reference signal. In this research, a single reference signal is assumed, although multiple signal can be used as in [13].

The $k$ th control filter $\mathbf{w}_{k}^{T}(n)$ is used to calculate $k$ th control signal, $u_{k}(n)$, obtained as follows:

$$
u_{k}(n)=\underset{6}{\mathbf{w}_{k}(n)^{T} \mathbf{x}_{u}(n)},
$$




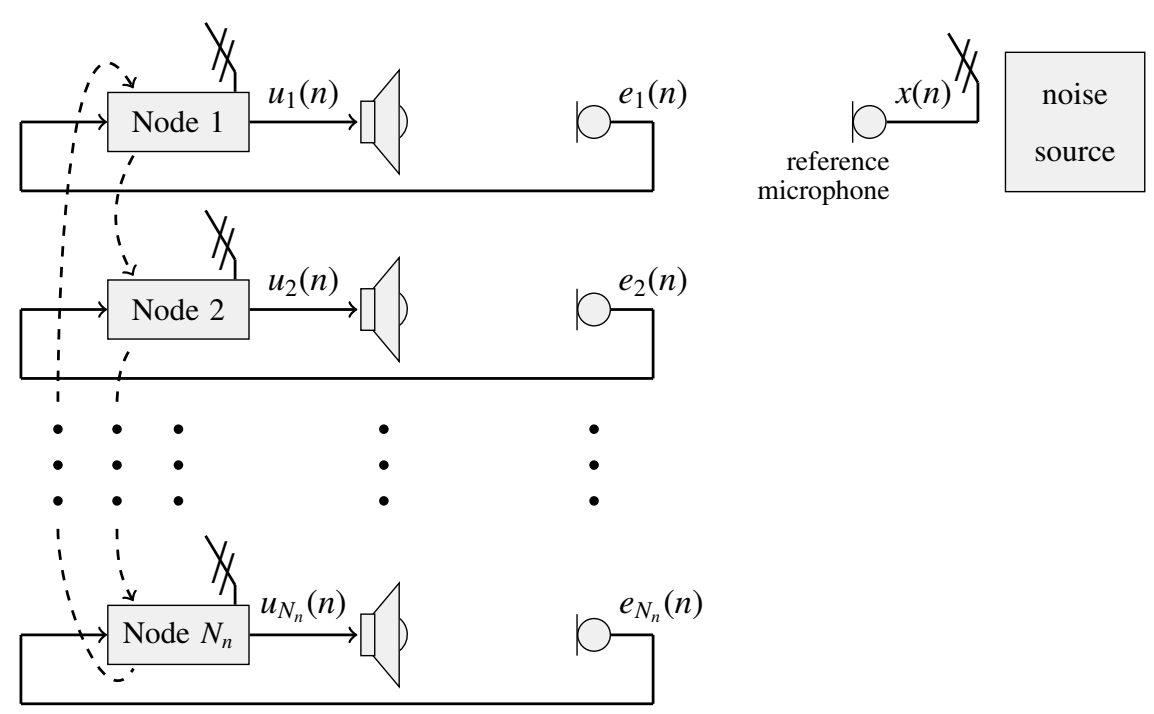

Figure 2: The schematic representation of distributed control system.

where $\mathbf{x}_{u}(n)=\left[x(n), x(n-1), \ldots, x\left(n-\left(L_{w}-1\right)\right)\right]^{T}$ is the vector of regressors of the reference signal.

In the distributed network, the $k$ th term of the sum of Eq. (1) is calculated by the $k$ th node. The $k$ th error signal $e_{k}(n)$ is obtained at the $k$ th microphone belonging to the $k$ th node. The secondary paths between all the loudspeakers and the $k$ th microphone, $\hat{\mathbf{s}}_{i k}$ for $i=1, \ldots, N_{n}$, are identified in the set-up stage and stored in the $k$ th node. The reference signal $x(n)$ is transmitted to all nodes through a separate communication channel.

To deal with a distributed processing, the local updating is performed following an incremental strategy [19]. For a given sample $n$, a complete round is performed along the network, where each node computes its term of the summation in Eq. 11, aggregates it to the given filter vector and passes it to the following node in an incremental order. The schematic representation of such operation is given in Fig. 2. Finally, once the global updated vector has been obtained at the last node, its values are disseminated to the rest of the nodes. It is justified to consider further reduction of the computational burden, as discussed in [20]. 


\subsubsection{Sensors and actuators arrangement}

Another important element of the ANC system is the set of sensors and actuators (typically microphones and loudspeakers). Their role is to measure, and then to appropriately alter the acoustic pressure in the surrounding environment (which is in general a three-dimensional space). But, their operation is rather limited to individual points in space. Obviously, an acoustic wave created at one point propagates through the acoustic field. However, the propagation is affected by absorption/reflection phenomena with walls and different objects in the acoustic environment, forming complicated patterns, what determine how particular loudspeakers or microphones interact with the surrounding acoustic field. Hence, the feasibility of certain control objectives (like generation of zones of quiet) depends on the sensors and actuators locations, and on the room acoustics as well. Therefore, the sensors and actuators arrangement should be carefully chosen, taking into account the defined goals. It would enable sensors to measure acoustic pressure changes in the target zones with satisfactory accuracy, as well as actuators to alter it based on the admissible energy effort [21].

\subsection{Problem formulation}

Performance of an Active Noise Control (ANC) system strongly depends on sensors and actuators spatial arrangement, determining both achieved Noise Reduction (NR) levels and spatial distribution of obtained zones of quiet. Also, there are often multiple constraints imposed on admissible sensors and actuators locations, making the problem complex and difficult to solve (e.g. loudspeakers may be impossible to be placed near the device emitting noise; also other obstacles may limit the admissible loudspeakers locations). However, if the acoustic environment of interest can be modelled (as it is in the considered scenario), then optimization algorithms can be employed to find efficient configuration of the ANC system, in order to enhance system performance.

In this research, it is assumed that the control algorithm is appropriately chosen and parametrized for the given application (it includes the adopted step size, control filters length, etc.) and no further considerations are provided on this issue. Therefore, the main remaining aspect that determines the successful operation of the ANC system is the sensors and actuators arrangement. As the performance measure, the Noise Reduction (NR) levels obtained in the target zones are used. 
Arrangement of sensors and actuators is described by their coordinates in space. In this research it is assumed that the coordinates take integer values (the domain is discrete), although, it can be easily changed to continuous domain if that is more convenient. The vector $\mathbf{x}_{a, i}$ of dimension $\left(D_{a} \times 1\right)$ contains coordinates describing location of the $i$ th actuator. On the other hand, the vector $\mathbf{x}_{s, i}$ of dimension $\left(D_{s} \times 1\right)$ contains coordinates describing location of the $i$ th sensor. The $D_{a}$ and $D_{s}$ determine the extent of spaces where actuators and sensors locations are defined, respectively.

To fully describe the arrangement and solve the undertaken problem, the optimal coordinates have to be found for all $N_{a}$ actuators and $N_{s}$ sensors. Hence, the total number of coordinates $N_{c}$ to find is defined by:

$$
N_{c}=D_{a} N_{a}+D_{s} N_{s} .
$$

Hence, the optimization algorithm have to find a solution in an $N_{c}$-dimensional space.

\section{Shaping spatial distribution of zones of quiet}

\subsection{An overview}

To provide a better overview of proposed method for shaping zones of quiet, the following steps can be distinguished:

1. Formulation of admissible ANC system configurations and control objective;

2. Selection of an active control algorithm and its parametrization;

3. Modelling of the acoustic environment (experimental or theoretical);

4. Optimization process according to the control objective;

5. Evaluation of the obtained results.

The first step, formulation of admissible ANC system configurations and control objective, is entirely application-dependent. The formulations follow from the problem description as, e.g., given in the previous Section. An example of such considerations is provided in Part II, where a particular scenario is analysed. The selection of an active control algorithm performed in the second step is out of the scope of this paper. For the purpose of this research, the control algorithm is arbitrarily chosen and its brief description is given in Subsection 2.2 The third step, modelling of the acoustic environment, is described in the following Subsection 3.2 It formulates the requirements for the model to be feasible and proposes a method to obtain it. The 
fourth and main step of the method, the optimization process, is also introduced in details in this Section. Firstly, several cost function are proposed in Subsection 3.3. Then, the optimization algorithm is described in Subsection 3.4 Within the fifth step a decision is made, whether the obtained results are satisfactory (if not, one or more of the previous steps may be re-evaluated and the optimization process run again).

\subsection{Modelling of the acoustic environment}

A proper model of the acoustic environment is required in order to provide the suitable spatial distribution of the quiet zones. It should represent acoustic paths between potential sound sources (admissible control loudspeakers' locations and, if it is possible, noise sources) and measurement points in the observation zone, i.e. part of the acoustic environment which will be modelled and where the ANC system will operate. Within the observation zone, the target zones will be defined, i.e. zones where the noise reduction is required. Most of the observation zone should be admissible for error microphones, but the observation zone may also include locations unavailable for error microphones in final configuration, but where the obtained NR levels are important and hence they should be taken into account during modelling. As a result, a matrix of acoustic paths models is obtained.

The model will be used to evaluate different configurations of the ANC system and to find the most efficient one. The computational complexity of the its processing may affect the simulation execution time, hence it should provide sufficient accuracy, preserving the simplicity. Therefore, the acoustic paths are modelled by using a FIR filter whose length is selected depending on the adopted sampling frequency and the reverberation time of the given acoustic environment. The FIR filter coefficients can be obtained both by theoretical calculations or experimental measurements (later approach is more recommended considering the complexity of most real environments). The measurements should obey all the rules of a proper system identification experiment, however, they are out of the scope of this paper (the reader is referred to, e.g., [22]).

An example of experimental measurements for the purpose of model identification is schematically presented in Fig. 3. The measurement process is divided into stages. The number of stages depends on the number of speakers and microphones available for simultaneous playback and recording, and the number of admissible locations to evaluate. In the first stage, employed microphones are places at first subset of admissible locations (see Fig. 3a). Then, the control speakers at appropriate locations playback a previously prepared set of orthogonal broadband 


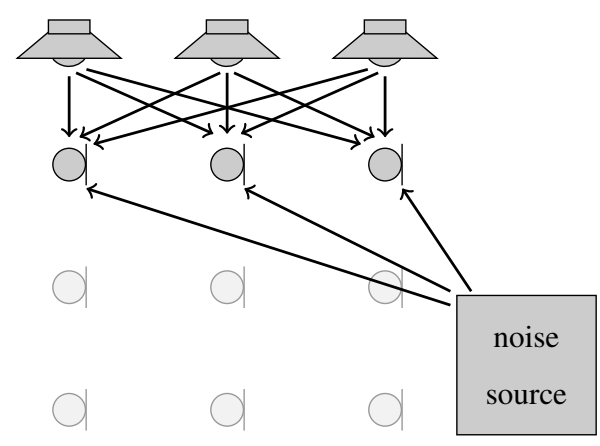

(a) Acoustic paths identified in stage 1 .

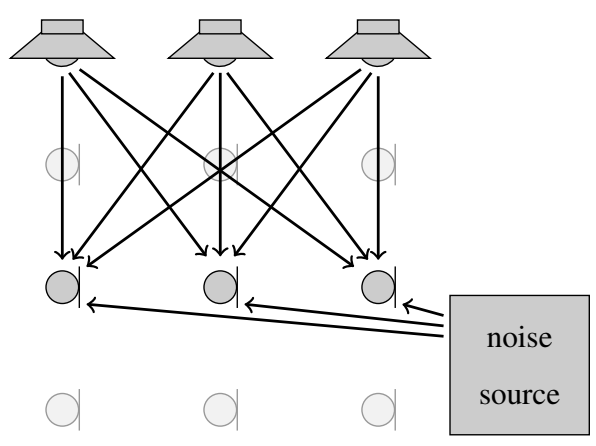

(b) Acoustic paths identified in stage 2 .

Figure 3: A visualization of model acoustic paths to identify (each arrow represent an identified acoustic path).

signals (or play the same signal individually, one by one, although it takes more time) and the recordings from all microphones are saved for to purpose of subsequent acoustic paths model identification. Afterwards, in the following stage, the microphones are moved to the next subset of admissible locations and the experiment is repeated (see Fig. 3b). Such procedure continues until all admissible microphone locations are evaluated. If the number of admissible loudspeakers locations exceeded the number of employed loudspeakers, then the loudspeakers are moved, and the whole procedure repeats. The process allows to identify acoustic paths between all admissible loudspeakers and microphones locations. Acoustic paths between the noise source and microphones can be identified in the same manner. In the described process, the paths for the reference microphone can be treated as for any other microphone.

\subsection{Cost functions}

The cost functions proposed in the described shaping method reflect the objective of generation of zones of quiet fitted to the precisely defined target zones of an arbitrary shape. The target zones are defined within boundaries of the observation zone. The first of proposed cost functions, $J_{1}$, reflects the aforementioned goals and is defined as:

$$
J_{1}=\frac{\sum_{\mathbf{x}} \mathrm{NR}(\mathbf{x}) \mathrm{A}_{1}(\mathbf{x})}{\sum_{\mathbf{x}} \mathrm{A}_{1}(\mathbf{x})},
$$

where $\mathrm{NR}(\mathbf{x})$ denotes the steady-state noise reduction level at point $\mathbf{x}$; the symbol $\mathbf{x}$ contains coordinates describing a point in the observation zone (the number of coordinates depends on 
number of dimensions of considered observation zone); $\mathrm{A}_{1}(\mathbf{x})$ is the shape function at position $\mathbf{x}$, representing the target zones shapes. In general, the shape function $\mathrm{A}_{1}(\mathbf{x})$ takes positive values for points where the noise reduction should be achieved and zeros for other points. The obtained zone of quiet may actually be wider and of an irregular shape, but at least in the target zones defined by the $\mathrm{A}_{1}(\mathbf{x})$ the noise reduction should be achieved.

The steady-state noise reduction level NR(x) is obtained by temporal average of the instantaneous noise reduction level $\mathrm{NR}(\mathbf{x}, n)$ at position $\mathbf{x}$ and time $n$, for the final part of the experiment, when the steady-state is reached:

$$
\mathrm{NR}(\mathbf{x})=\frac{\sum_{n=(1-p) N_{\text {exp }}}^{N_{\text {exp }}} \mathrm{NR}(\mathbf{x}, n)}{p N_{\text {exp }}},
$$

where $N_{\text {exp }}$ is the number of samples generated in an experiment, and $0<p \leq 1$ is a real number denoting the fraction of the experiment when a steady-state is reached. The $\mathrm{NR}(\mathbf{x}, n)$ is obtained as the ratio in $\mathrm{dB}$ between the instantaneous estimated error power with and without the application of the ANC system:

$$
\mathrm{NR}(\mathbf{x}, n)=10 \log _{10}\left[\frac{e^{2}(\mathbf{x}, n)}{d^{2}(\mathbf{x}, n)}\right],
$$

where $e(\mathbf{x}, n)$ and $d(\mathbf{x}, n)$ are the error signal and the primary disturbance signal, respectively, at position $\mathbf{x}$ and time $n$. Negative values indicate a noise reduction, while positive values represent noise enhancement. Hence, the aim is to minimize the cost functions.

The cost function presented in Eq. (5) can be extended to take into account sound pressure spatial gradient. It should be minimized in order to avoid unpleasant sudden changes of experienced sound pressure while walking in the room (e.g., entering and exiting the target zones). Such cost function, $J_{2}$, can be expressed as:

$$
J_{2}=\frac{\sum_{\mathbf{x}} \mathrm{NR}(\mathbf{x}) \mathrm{A}_{1}(\mathbf{x})}{\sum_{\mathbf{x}} \mathrm{A}_{1}(\mathbf{x})}+a_{2} \frac{\sum_{\mathbf{x}}\|\nabla \mathrm{E}(\mathbf{x})\| \mathrm{A}_{2}(\mathbf{x})}{\sum_{\mathbf{x}} \mathrm{A}_{2}(\mathbf{x})},
$$

where $\|\cdot\|$ denotes the Euclidean norm, $\nabla(\cdot)$ is a gradient operator and $\mathrm{A}_{2}(\mathbf{x})$ is the shape function representing the zone where sound pressure spatial gradient is taken into account (if the spatial gradient should be considered in the whole observation zone, then $\mathrm{A}_{2}(\mathbf{x})=1$ for all $\left.\mathbf{x}\right)$. $a_{2}$ is a weighting parameter between the two goals: noise reduction and minimization of sound pressure spatial gradient. $\mathrm{E}(\mathbf{x})$ is energy estimator of error signal at point $\mathbf{x}$ at steady state. $\mathrm{E}(\mathbf{x})$ 
is calculated by temporal average of $\mathrm{E}(\mathbf{x}, n)$ as:

$$
\mathrm{E}(\mathbf{x})=\frac{\sum_{n=(1-p) N_{\text {exp }}}^{N_{\text {exp }}} \mathrm{E}(\mathbf{x}, n)}{p N_{\text {exp }}},
$$

where $\mathrm{E}(\mathbf{x}, n)$ is an instantaneous energy estimator of error signal at point $\mathbf{x}$ and sample $n$. It is calculated by:

$$
\mathrm{E}(\mathbf{x}, n)=10 \log _{10}\left[e^{2}(\mathbf{x}, n)\right] .
$$

Alternatively, depending on the considered application, in Eq. (8) the gradient of noise reduction level $\nabla N R(\mathbf{x})$ can be used, instead of $\nabla \mathrm{E}(\mathbf{x})$.

If a non-stationary primary disturbance field is considered, then simulations can be performed for a given representative recording of the non-stationary primary noise. In such a case, performance of ANC system can be evaluated not only by terms of steady-state NR levels, but also by the behaviour in the transient states. The ANC system should adapt quickly to new conditions without unwanted noise enhancement in the transient state. One of the possible approaches is to include temporal derivative of sound pressure into cost function to penalize rapid and unexpected changes in time of sound pressure (due to, e.g., slow adaptation or too rapid reaction of ANC system to changing conditions). Then, the cost function, $J_{3}$, takes form:

$$
J_{3}=\frac{\sum_{\mathbf{x}} \frac{1}{N_{\text {exp }}} \sum_{n=1}^{N_{\text {exp }}} \mathrm{NR}(\mathbf{x}, n) \mathrm{A}_{1}(\mathbf{x})}{\sum_{\mathbf{x}} \mathrm{A}_{1}(\mathbf{x})}+a_{3} \frac{\sum_{\mathbf{x}} \frac{1}{N_{\text {exp }}} \sum_{n=1}^{N_{\text {exp }}} \dot{\mathrm{E}}(\mathbf{x}, n) \mathrm{A}_{3}(\mathbf{x})}{\sum_{\mathbf{x}} \mathrm{A}_{3}(\mathbf{x})}
$$

where superimposed dot denotes the temporal derivative; $\mathrm{A}_{3}(\mathbf{x})$ is the shape function representing the zone where sound pressure temporal derivative is taken into account; $a_{3}$ is a weighting parameter describing the importance of minimization of sound pressure temporal derivative. Moving average or other filter of similar purpose can be applied on $\mathrm{E}(\mathbf{x}, n)$ signal before calculating the temporal derivative.

The minimization of sound pressure spatial gradient as in 87 , can also be incorporated into cost function defined in 11], resulting in:

$$
J_{4}=\frac{\sum_{\mathbf{x}} \frac{1}{N_{\text {exp }}} \sum_{n=1}^{N_{\text {exp }}} \mathrm{NR}(\mathbf{x}, n) \mathrm{A}_{1}(\mathbf{x})}{\sum_{\mathbf{x}} \mathrm{A}_{1}(\mathbf{x})}+a_{2} \frac{\sum_{\mathbf{x}} \frac{1}{N_{\text {exp }}} \sum_{n=1}^{N_{\text {exp }}}\|\nabla \mathrm{E}(\mathbf{x}, n)\| \mathrm{A}_{2}(\mathbf{x})}{\sum_{\mathbf{x}} \mathrm{A}_{2}(\mathbf{x})}+a_{3} \frac{\sum_{\mathbf{x}} \frac{1}{N_{\text {exp }}} \sum_{n=1}^{N_{\text {exp }}} \dot{\mathrm{E}}(\mathbf{x}, n) \mathrm{A}_{3}(\mathbf{x})}{\sum_{\mathbf{x}} \mathrm{A}_{3}(\mathbf{x})} .
$$


Worth mentioning is also the possibility to alter the signals $\mathrm{NR}(\mathbf{x}, n)$ and/or $\mathrm{E}(\mathbf{x}, n)$ in frequency domain before providing them to one of the proposed cost functions (e.g., by filtering). It would enable to shape the obtained frequency characteristics in a desired manner.

\subsection{Optimization algorithm}

The search spaces followed from the problem described in the previous section are very complicated. An efficient algorithm have to be employed in the optimization process to find a solution satisfying defined demands. Evolutionary algorithms have been proven to be a versatile and effective technique for solving nonlinear optimization problems with multiple optima [23]. However, they usually require evaluation of numerous solutions resulting in high computational cost. To mitigate this drawback, a Memetic Algorithm (MA) can be utilised, which is a hybrid form of population-based approach coupled with separate individual learning. The MA combines advantages of a global search, like for evolutionary algorithms, and local refinement procedures, which enhance converge to the local optima [24]. Due to such complementary properties they are particularly useful in solving complex multi-parameter optimization problems, such as placement of sensor and actuators [25].

A flowchart of the employed memetic algorithm is presented in Fig. 4 The algorithm starts with the initialization step, where a given number of individuals (potential solutions) is randomly generated to create the initial population (a pool of currently considered solutions). In the evaluation step, the fitness function is calculated for each individual in the population. It is the part of the algorithm, which consumes most of the computation time, as it requires a complete ANC system simulation for each fitness function evaluation. Hence, parallelized computation is advisable here. Afterwards, the individuals are sorted based on the obtained fitness values. Then, the termination criteria are checked-to find out whether the fixed maximum number of generations is reached or a convergence is detected (a number of recent generations resulted in no improvement). If any of them is met, then the process is finished. Otherwise, it continues and the selection is performed, where a part of the existing population is chosen for reproduction dependent on the fitness value. A fixed number of the fittest individuals is selected (survive) and the rest is rejected (die out). Then, in the crossover step, children solutions are generated by applying a crossover operator for a number of selected pairs of individuals (parents). Individuals fitting better are more likely to be chosen as parents. Children join the population not replacing the parents, yet. It may happen later, but dependent on their fitness what will be resolved through 


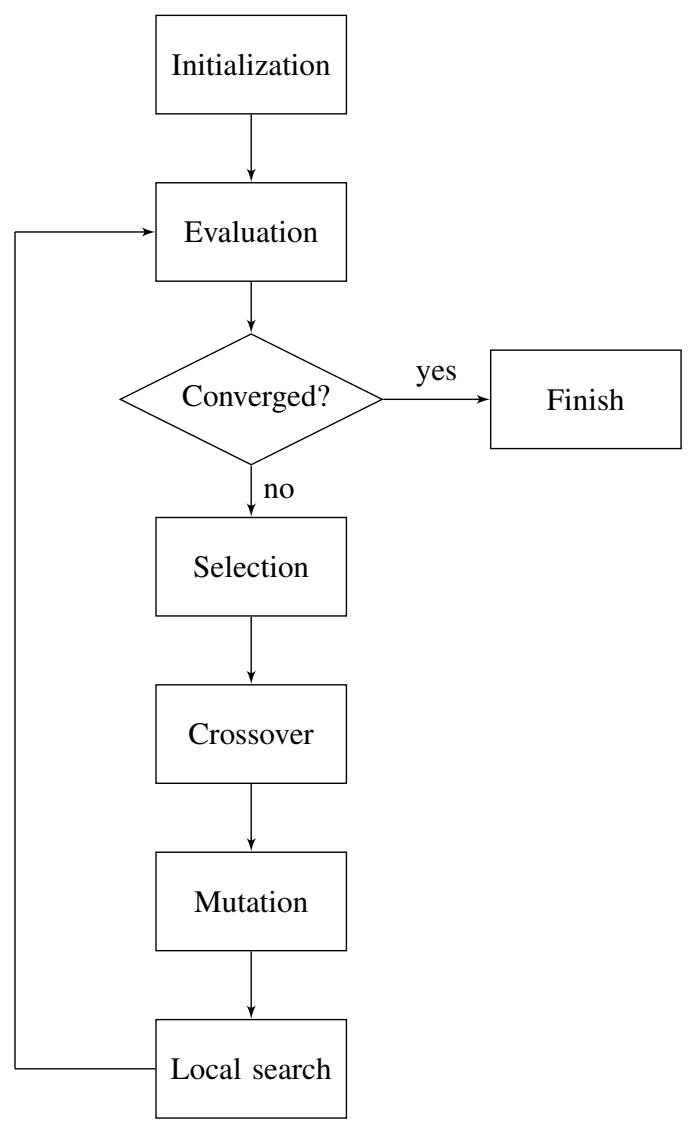

Figure 4: A memetic algorithm flowchart.

the evaluation and selection steps. It is equivalent to the elitist selection strategy, which allows a limited number of the best parents to carry over to the next generation, unaltered. It is a practical approach, because it ensures that the solution quality do not decrease from one generation to the next. To maintain genetic diversity (counteract premature convergence and ensure global search), the mutation operator is used in the mutation step. The operator is applied dependent on a predefined probability. In this implementation, the mutation results in creation of an additional individual joining the population, keeping the original individual unaltered. Then, a local search operator is employed to improve individual fitness (the local search step). To maintain a balance between the degree of evolution (exploration) and individual improvement (exploitation), only a portion of the population individuals undergo the learning. Afterwards, a evaluation is performed, and the process is repeated until the termination criteria is met. 


\subsection{Problem representation in the $M A$}

The goal of the optimization is to find optimal arrangement of sensors and actuators, respecting only the admissible locations and other imposed constraints. Hence, a particular individual in the MA represents a particular configuration of the ANC system. It consists of $N_{c}$ coordinates of all the sensors ( $D_{s}$ coordinates per sensor) and actuators $\left(D_{a}\right.$ coordinate per actuator). To provide an example, let us assume that the coordinates are integer and that $D_{a}=1$ and $D_{s}=2$ (the actuators location search space is one-dimensional and the microphone search space is two-dimensional). Let us also adopt, similarly as in [13], that the number of nodes of the control system equals $N_{n}$, where each node consists of one sensor and one actuator. Therefore one node is described by $D_{a}+D_{s}=3$ coordinates, and whole system is described in total by $N_{c}=3 N_{n}$ coordinates. A visualization of an exemplary individual's vector of coordinates is given in Fig. 5. The $x_{s, i, j}$ and $x_{a, i, j}$ represents $j$ th coordinate of $i$ th sensor and actuator, respectively.

\begin{tabular}{|c|c|c|c|c|c|c|c|c|c|}
\hline \multirow{3}{*}{ individual } & \multicolumn{3}{|c|}{ node no. 1} & & & & \multicolumn{3}{|c|}{ node no. 3} \\
\hline & $x_{a, 1,1}$ & $x_{s, 1,1}$ & $x_{s, 1,2}$ & \multicolumn{3}{|c|}{ node no. 2} & $x_{a, 3,1}$ & $x_{s, 3,1}$ & $x_{s, 3,2}$ \\
\hline & 4 & 5 & & \multirow{2}{*}{$\begin{array}{c}x_{a, 2,1} \\
65\end{array}$} & \multirow{2}{*}{$\begin{array}{c}x_{s, 2,1} \\
8\end{array}$} & \multirow{2}{*}{$\begin{array}{c}x_{s, 2,2} \\
8\end{array}$} & 16 & & 8 \\
\hline & & & & & & & & & \\
\hline
\end{tabular}

Figure 5: An exemplary individual's visualization for $N_{n}=3$.

\subsection{Operators}

In this Subsection operators employed in the MA are described. The greatest attention is given to the application-specific features.

\subsubsection{Crossover operator}

The crossover operator utilizes two selected individuals (parents) to create another individual (conceive a child). A visualization of an exemplary crossover operation is presented in Fig. 6 The general concept is that the child should inherit features of the parents, however, a particular method to effectively achieve this goal is very application-specific. In the discussed implementation, every individual has always the same fixed number of nodes $N_{n}$, hence, there are $N_{n}$ nodes to be defined (inherited) for a child, based on $2 N_{n}$ nodes belonging in total to parents. For the $i$ th node of a child, the loudspeaker coordinates $\mathbf{x}_{a, i}$ and the microphone coordinates $\mathbf{x}_{s, i}$ are separately inherited (the node is not inherited as a whole in the crossover). Hence, e.g. a particular 


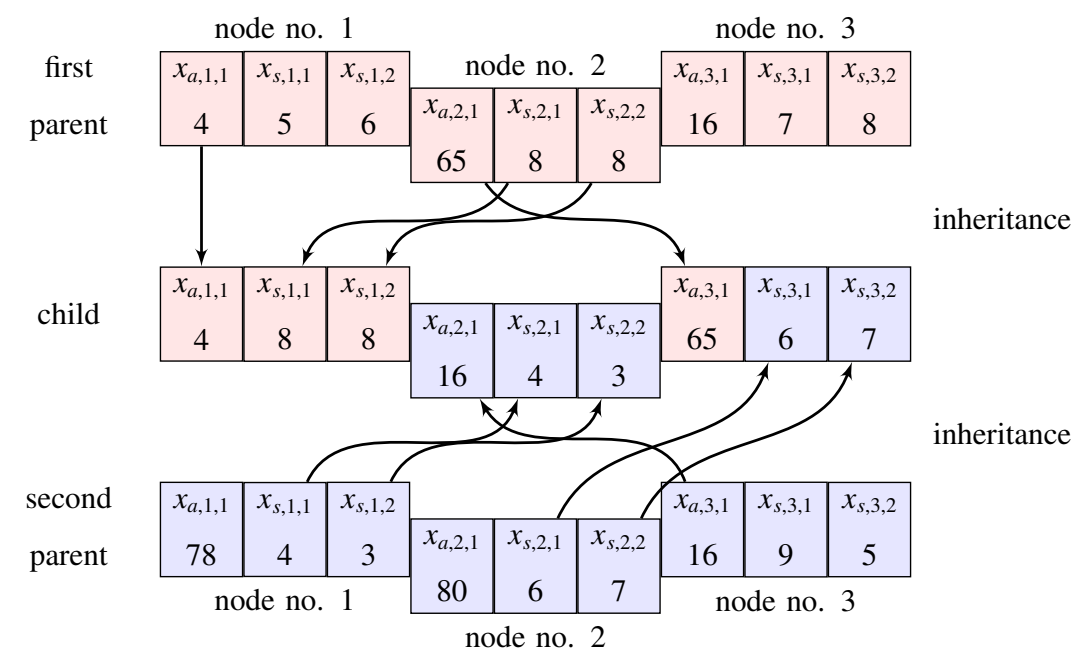

Figure 6: An exemplary crossover operation visualization for $N_{n}=3$.

loudspeaker coordinates $\mathbf{x}_{a, i}$ can be inherited from any randomly-selected node of the first or the second parent. It means that for a particular node, a loudspeaker location can be inherited from one parent and the microphone location can be inherited from the other parent. Such approach introduces more randomness in the crossover operation, than in a strategy, where nodes would be considered as a whole, and it has been observed that it results in a faster convergence of the MA.

Using the described crossover operator implementation, it is plausible that a child would inherit several times the same coordinates for loudspeakers in different nodes. It is impossible to place several loudspeakers at exactly the same location. However, to avoid rejecting such individual entirely as inadmissible, a "repairing procedure" is initialized. Namely, the overlapping loudspeakers are distinguished and one of them is moved to a neighbouring location. On the other hand, overlapping microphones of several nodes are allowed and it is interpreted as nodes sharing a common error signal from actually one microphone.

\subsubsection{Mutation operator}

The mutation operator in each generation is applied to a portion of population. The individuals are randomly selected. When applied to an individual, one or more of its coordinates (belonging to loudspeakers or microphones) are changed to a random value. In a single generation the mutation operator can be applied many times to the same individual. Each time an additional mutated individual joins the population, keeping the original individual unaltered. The "repairing 
procedure" described earlier is also applied if necessary.

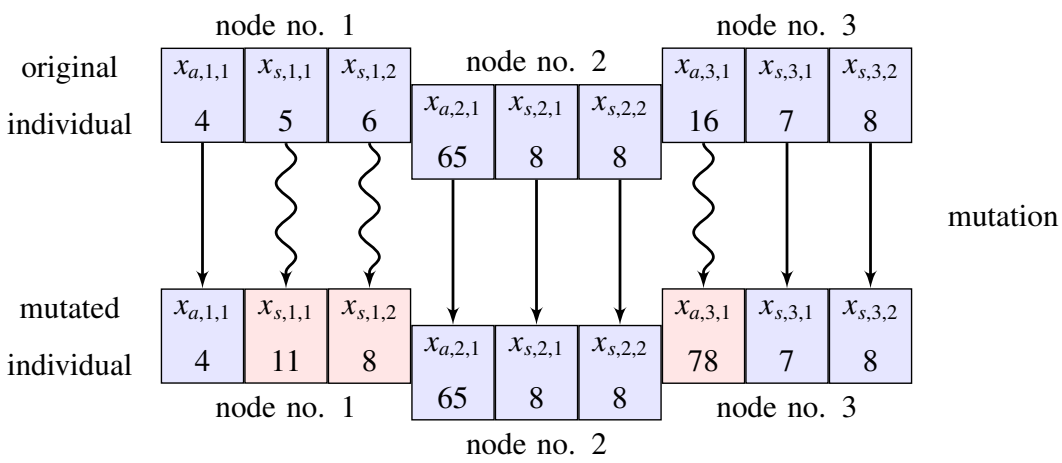

Figure 7: An exemplary mutation operation visualization for $N_{n}=3$.

\subsubsection{Local search operator}

In this implementation, the "Hill climbing" technique [24] is chosen as the individual learning strategy. In this strategy, for an affected individual a neighbour is created - a clone of the original individual with one of coordinates slightly changed (a location of a loudspeaker or microphones is moved by a short distance in one of the admissible directions). Then, the fitness of the original individual and the neighbour is compared. If the neighbour is better, it replaces the original individual. Otherwise, it is discarded. Afterwards, another neighbour is generated and the procedure repeats. The process continues until a fixed number of following generated neighbours are worse than the original individual. If so, it is assumed that a local optimum has been reached and the local search operator finishes.

\section{Simulation studies}

\subsection{The employed scenario}

The presented simulation studies employ an exemplary scenario that has been introduced in Subsection 2.1. In the role of the described environment, a real room located at the Audio Processing Laboratory of the Polytechnic University of Valencia has been utilized (a photograph of the listening room is shown in Fig. 8). It is a listening room of $9.36 \mathrm{~m}$ long by $4.78 \mathrm{~m}$ wide by $2.63 \mathrm{~m}$ high. The room is equipped with an array of 96 independently driven loudspeakers deployed in an octagonal shape (thus the space of admissible actuators locations is conveniently 


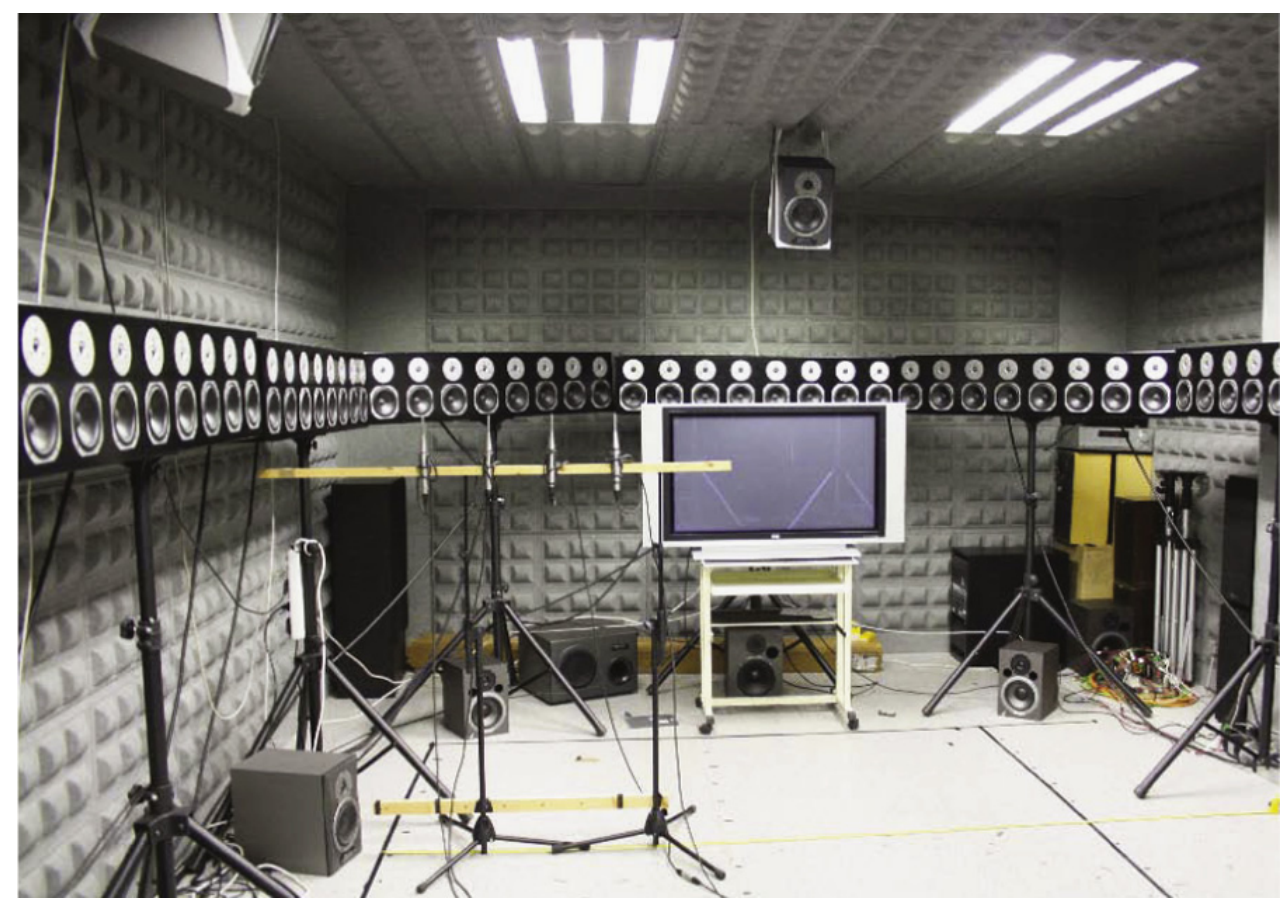

Figure 8: A photograph of the listening room at the Audio Processing Laboratory of the Polytechnic University of Valencia.

described by number of dimensions $\left.D_{a}=1\right)$. The two-dimensional observation zone $(4.6 \mathrm{~m}$ long and $3.0 \mathrm{~m}$ wide, and at the height of $1.47 \mathrm{~m}$ ), where the acoustic pressure is monitored, is enclosed within the loudspeakers array (a schematic representation of the room is given in Fig.9). The area has been divided to obtain a grid of 24 by 16 points (the interval between adjacent points is $0.2 \mathrm{~m}$ ), where the acoustic responses due to excitation generated by each speaker have been measured. The ANC system sensors can be virtually placed anywhere in the observation zone, hence, the space where sensors locations are defined posses the number of dimensions $D_{s}=2$. The responses are modelled as FIR filters of 256 coefficients ( $2 \mathrm{kHz}$ sampling frequency is used, as ANC methods are mostly employed for low frequencies). It resulted in identification of 36864 acoustic responses in total. Such set constitutes a model that enables simulation of ANC systems with loudspeakers located at any of the 96 locations and microphones at any of 384 locations. It also enables a simulation of acoustic pressure in the whole observation zone (not only at locations where the ANC system error microphones are placed), what constitutes a great value in evaluation and understanding of the overall behaviour of the control system. To 
Shaping zones of quiet generated by an active noise control system

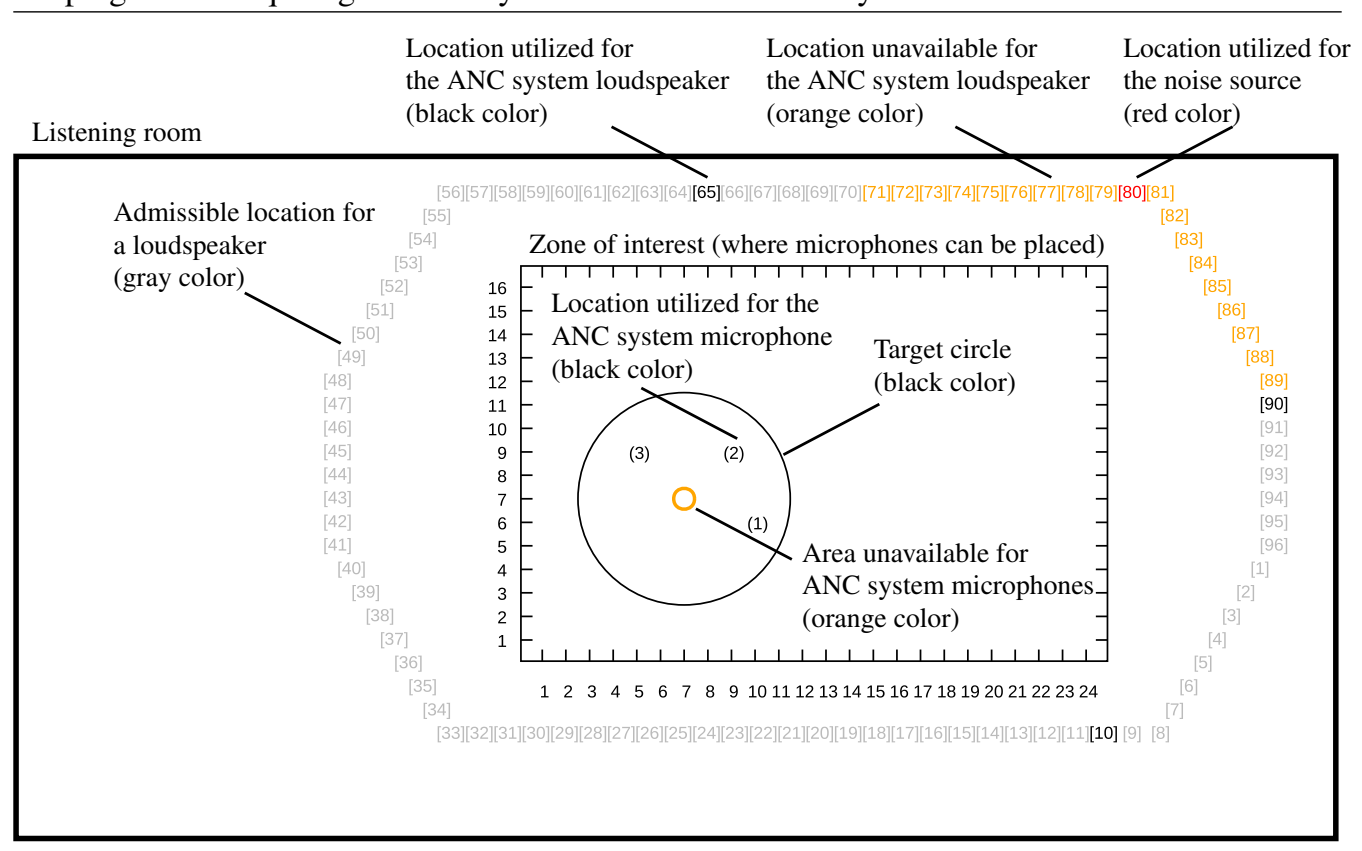

Figure 9: A schematic of the listening room.

simulate the noise source, one of the loudspeakers potential locations is used. In the employed scenario, the primary noise source (representing the device emitting a broadband noise) is located at 80th loudspeaker position (it is marked with red colour in Fig. 9). It is a randomly selected location without any specific properties. However, to facilitate any further comparison between scenarios, this location is kept constant. On the other hand, location of the user is chosen to be at coordinates $(7,7)$ in the observation zone. Once again, this location does not represent any particular acoustic properties, however, it was manually selected to keep a distance from the observation zone boundaries, in order to monitor generated zones of quiet. The user location is also kept constant through the scenarios.

It is also assumed that ANC system loudspeakers cannot be placed at the location already taken by any other loudspeaker (ANC system actuators or the noise source). Moreover, ANC system loudspeakers often need to keep some distance from the device generating noise (due to e.g., large dimensions of the device itself, supporting devices or installations occupying space around the device, or other technical reasons). To represent such situations in considered scenarios, a margin from the noise source $M_{s}$ is introduced. It takes integer values greater or equal one, denoting minimal admissible distance between the noise source (at position 80th) and the nearest 
ANC loudspeaker. An example of $M_{s}=10$ is visible in Fig. 9, where unavailable loudspeaker locations are marked with orange colour and the nearest admissible locations are 70th or 90th position. On the other hand, microphones are allowed to overlay each other (it is then interpreted as actually one microphone, which provides signal to more than one channel). However, it is rarely experienced, as it provides less information and affects system performance. Also, analogously as with the margin from the noise source $M_{s}$, a margin from the user location $M_{c}$ is introduced. It takes integer values greater or equal zero, denoting minimal admissible distance between the user location (at coordinates $(7,7)$ in the observation zone) and the nearest microphone. It represents an area in the observation zone, which is occupied by the user and his equipment, making it unavailable for ANC system microphones. An example of $M_{c}=1$ is given in Fig. 9, where the unavailable area is marked with the orange circle. It represents only a small margin, reserving minimal space for the user.

As a result of ANC system operations, zones of quiet are generated. Employing the proposed method of shaping spatial distribution of zones of quiet, they can be precisely defined. In this research, circular target zones are designed, introducing a target circle where the noise reduction is demanded. The circle is described by the coordinates of its centre $c_{x}$ and $c_{y}$, and radius $c_{r}$. The obtained zone of quiet may actually be wider and of an irregular shape, but at least in the target circle the noise reduction should be achieved. Target circle centre should be placed at the user location, therefore $c_{x}=7$ and $c_{y}=7$ in all scenarios. The target circle radius $c_{r}$ may vary. The target circle is represented in the schematic in Fig. 9 by a black circle. A cost function reflecting such objective has been given in Eq. [5], where:

$$
\mathbf{x}=[x, y]^{T},
$$

for:

$$
\begin{aligned}
& x \in\left\{1,2, \ldots, x_{\max }\right\}, \\
& y \in\left\{1,2, \ldots, y_{\max }\right\},
\end{aligned}
$$

In Eq. (14), $x_{\max }$ and $y_{\max }$ are bounds of the observation zone in the $x$ and $y$ directions, respectively. In the employed scenario, $x_{\max }=24$ and $y_{\max }=16$. The shape function $\mathrm{A}(x, y)$ is thus defined:

$$
\mathrm{A}(x, y)=\left\{\begin{array}{cc}
1, & \left(x-c_{x}\right)^{2}+\left(y-c_{y}\right)^{2} \leq c_{r}{ }^{2}, \\
0, & \left(x-c_{x}\right)^{2}+\left(y-c_{y}\right)^{2}>c_{r}{ }^{2} .
\end{array}\right.
$$




\subsection{The ANC system}

In the presented simulation studies, it is assumed that the control algorithm is appropriately parametrized for the given application. The length of control filters is $L_{w}=512$, the length of secondary paths models is $L_{s}=256$, the step size $\mu=0.0001$, and leakage coefficient is $\alpha=$ 0.999999. No further considerations are provided on this issue. Therefore, the main remaining aspect that determines the successful operation of the ANC system is the sensors and actuators arrangement.

\subsection{Optimization algorithm}

The configuration of the MA was kept the same through all scenarios. Three most important parameters for the MA performance are the crossover probability, mutation probability, and local search probability-all of them were set to $5 \%$. These values have been obtained based on preliminary experiments. The maximum number of generations was set to 24 , however, due to convergence detection (when five recent generations resulted in no improvement), often the optimization process was terminated earlier. In the local search operator, the stop condition (a number of following generated neighbours worse than the original individual) was set to 16 . On the other hand, the population quantity after the selection step was set to 500 (the excessive and less fitted individuals are rejected—die out).

\section{Simulation results}

In this Section, results on the shaping of the spatial distribution of the zones of quiet are presented. The ANC system control results are obtained by simulation of the algorithm with the identified acoustic paths.

Firstly, an individual experiment is presented in details to give a better insight into the performed simulations. Temporal, spatial and frequency characteristics are given. Subsequently, three important variants of the scenario are evaluated, studying influence of three important parameters on the obtained noise reduction results: margins $M_{c}, M_{s}$, and radius $c_{r}$. The studies are presented mainly on the basis of spatial characteristics in a steady-state stage and values of optimization index $J_{1}$ defined in Eq. [5]. 
Shaping zones of quiet generated by an active noise control system

\subsection{Example of an individual experiment}

To present performance of the ANC system, three main signals are evaluated: primary disturbance signal $d(x, y, n)$; error signal $e(x, y, n)$; and noise reduction $\mathrm{NR}(x, y, n)$. In this research, the $d(x, y, n)$ signal is a stationary low-frequency broadband random noise, concentrated mainly in the range from $50 \mathrm{~Hz}$ to $250 \mathrm{~Hz}$. It is exactly repeated in each simulation to facilitate a fair comparison between different configurations of the ANC system. On the other hand, the error

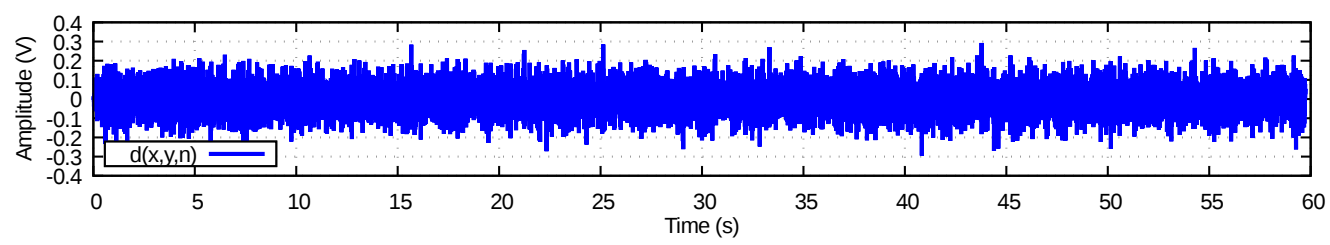

(a) Time plot of the primary disturbance signal $d(x, y, n)$ for $x=x_{s, 1}$ and $y=y_{s, 1}$.

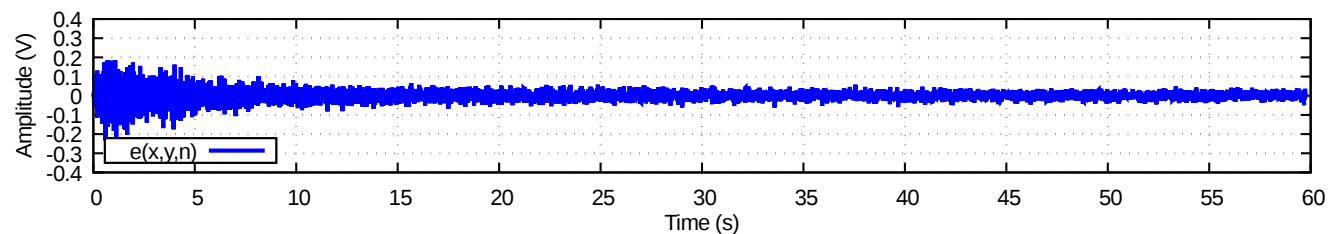

(b) Time plot of the error signal $e(x, y, n)$ for $x=x_{s, 1}$ and $y=y_{s, 1}$.

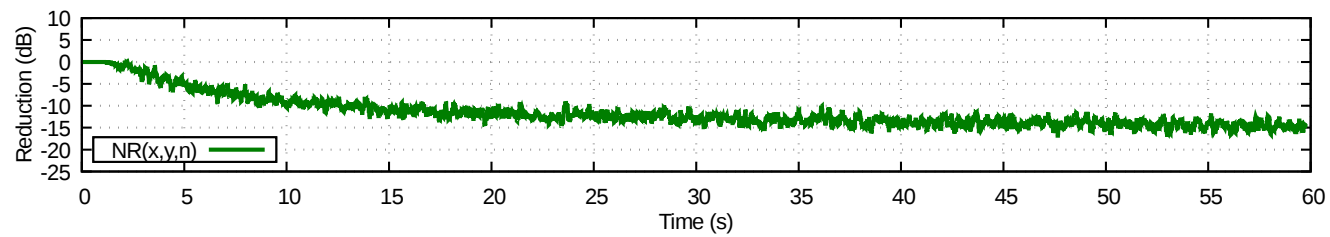

(c) Time plot of the noise reduction level $\operatorname{NR}(x, y, n)$ for $x=x_{s, 1}$ and $y=y_{s, 1}$.

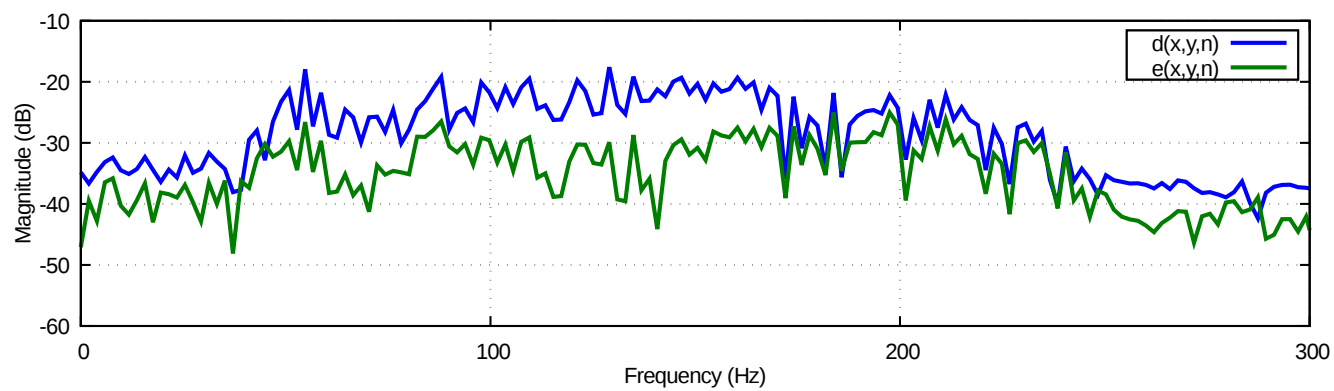

(d) Frequency characteristics of the primary disturbance signal $d(x, y, n)$ and error signal $e(x, y, n)$ in the final stage of the experiment.

Figure 10: Time plots of primary disturbance signal, error signal, and noise reduction level obtained for the first node. Frequency characteristics are also given. The presented individual experiment has been performed for $N_{n}=3, c_{x}=7$, $c_{y}=7, c_{r}=3, M_{c}=1$, and $M_{s}=10$. 


\section{Shaping zones of quiet generated by an active noise control system}

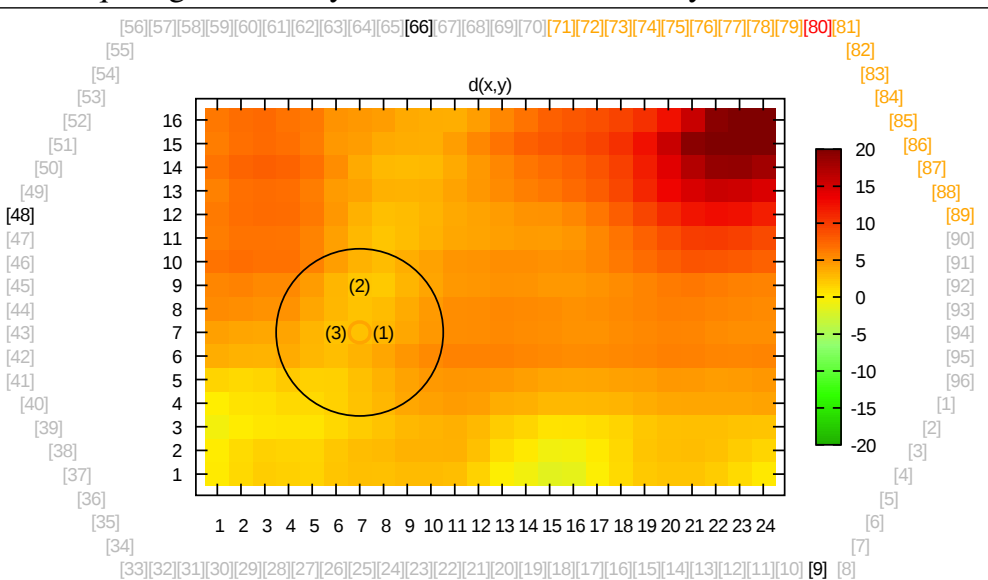

(a) Spatial distributions of the steady-state primary disturbance signal $d(x, y)$, given in $\mathrm{dB}$.

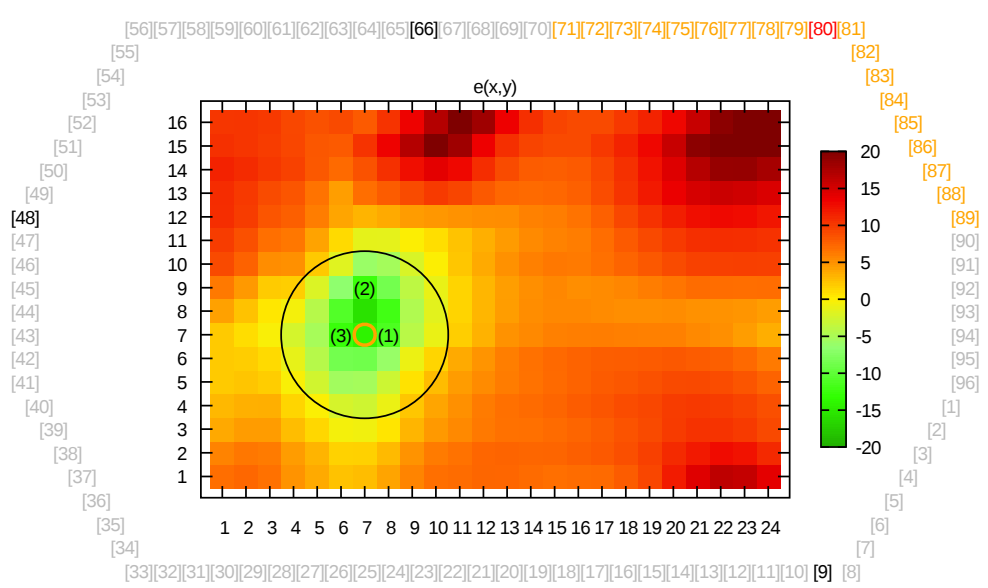

(b) Spatial distributions of the steady-state error signal $e(x, y)$, given in $\mathrm{dB}$

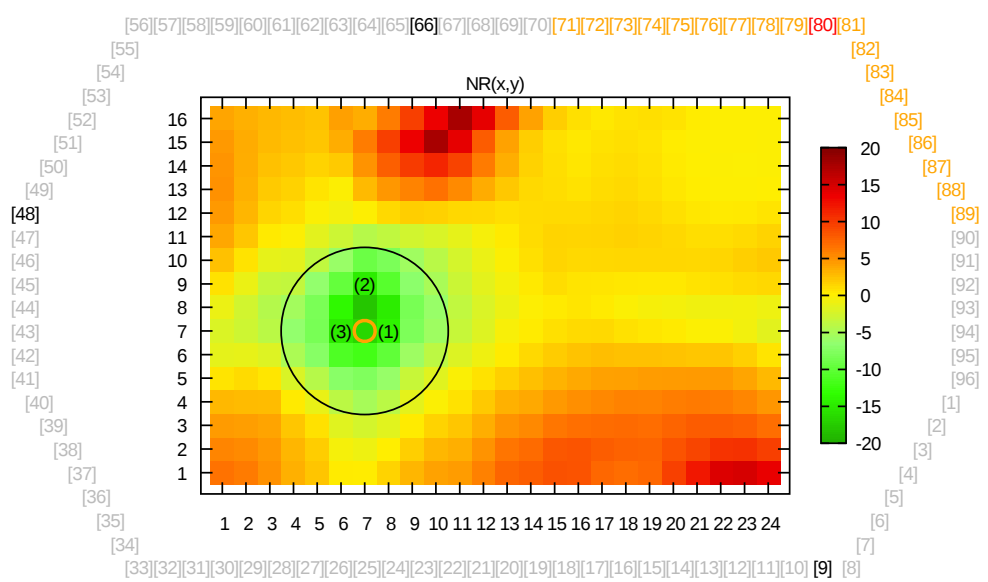

(c) Spatial distributions of the steady-state noise reduction level $\mathrm{NR}(x, y)$, given in $\mathrm{dB}$.

Figure 11: Spatial distribution charts of primary disturbance signal, error signal, and noise reduction level in the steadystate. The presented individual experiment has been performed for $N_{n}=3, c_{x}=7, c_{y}=7, c_{r}=3, M_{c}=1$, and $M_{s}=10$. The black and orange circles represents the target circle and the margin from the target circle centre $M_{c}$, respectively. 
signal $e(x, y, n)$ is a result of superposition of the primary disturbance and control signals generated by the ANC system. It is what the user (operator) of the device will actually hear. However, to better notice the difference that the ANC system makes, the noise reduction $\mathrm{NR}(x, y, n)$ is introduced, defined by Eq. (7).

An exemplary experiment is presented here, with the ANC system consisting of $N_{n}=3$ nodes. The goal was to generate a circular zone of quiet, with $c_{x}=7, c_{y}=7$, and $c_{r}=3$. The radius $c_{r}=3$ in the adopted coordinates system results in a circle of $1.2 \mathrm{~m}$ in the diameter. The margin values have been adopted as $M_{c}=1$, and $M_{s}=10$, reserving only the centre of the target circle for the user's head, and forcing a distance from the device to the nearest loudspeaker of at least $2 \mathrm{~m}$. With such parameters, the proposed method for shaping the zones of quiet spatial distribution has been used, as described in the Part I. As a result, an arrangement of three control loudspeakers and three error microphones have been obtained.

Temporal and frequency characteristics of the aforementioned signals obtained in an exemplary individual experiment are shown in Fig. 10 . It is assumed that the final 5\% of the experiment duration can be considered as the steady state. Therefore, spatial characteristics of the considered signals at steady state are presented in Fig. 11 It follows from the analysis of the Fig. $11 \mathrm{c}$ that the sound level has been enhanced in the proximity of control loudspeakers. However, it does not undermine the main goal, because in the target circle the noise has been reduced, resulting in a optimization index $J_{1}=-9.5$, what means that the average noise reduction in the target circle exceeded $9 \mathrm{~dB}$. It can be considered as a good result.

As the main quantity to assess the ANC system performance, the NR values are chosen. Hence, for the sake of brevity, in following subsections spatial distribution of the $\operatorname{NR}(x, y)$ only will be presented. 


\subsection{Margin around the noise source}

An impact of the margin from the noise source $M_{s}$ values on the noise reduction performance are evaluated in this Subsection. The $M_{s}$ parameter takes integer values greater or equal to one, reflecting minimal distance between the noise source and the nearest ANC loudspeaker. A series of evaluations of the proposed shaping method has been performed for different values of $M_{s}$ in the range from 1 to 30 (where $M_{s}=30$ means that more than a half of admissible positions have been reserved). Different number of nodes has also been tested. The other parameters take constant values: $c_{x}=7, c_{y}=7, c_{r}=4$, and $M_{c}=1$. Resulting relation between optimization index $J$, margin $M_{s}$, and number of nodes $N_{n}$ is presented in Fig. 12. In addition, for three selected values of the $M_{s}$ (equal 1,4 and 8 , respectively) spatial distributions of $\mathrm{NR}(x, y)$ are presented in Fig. 13 .

It follows from the analysis of Figs. 12 13 that three main stages can be distinguished. First, is a stage when value of $M_{s}$ is minimal. Then, a very convenient situation (and also rare in practice - real implementation of this configuration presents causality difficulties) occurs, when control loudspeakers can take places almost in the same spot where the noise source is. It results in very similar primary and secondary paths, and enables a global noise reduction (visible in Fig. 13a). It is also noteworthy that the microphones' locations are less important in such a case

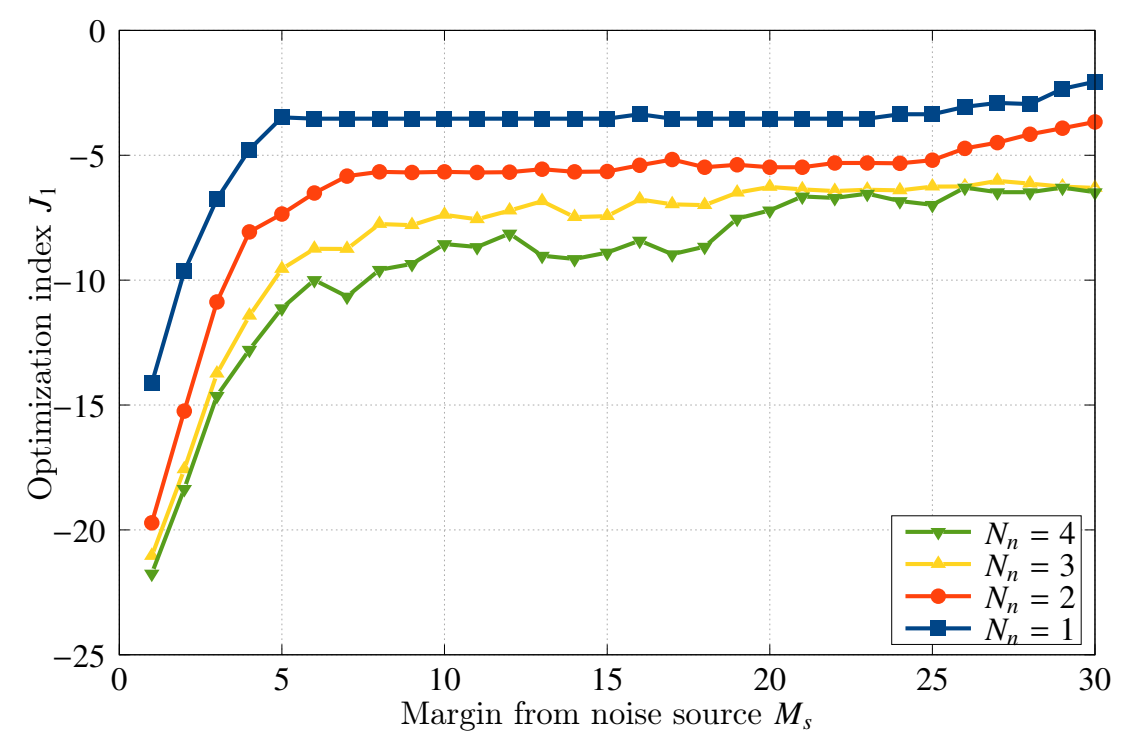

Figure 12: A plot representing relationships between the margin from noise source $M_{s}$, the number of nodes $N_{n}$, and the optimization index $J$. The experiments has been performed for $c_{x}=7, c_{y}=7, c_{r}=4$, and $M_{c}=1$. 
Shaping zones of quiet generated by an active noise control system

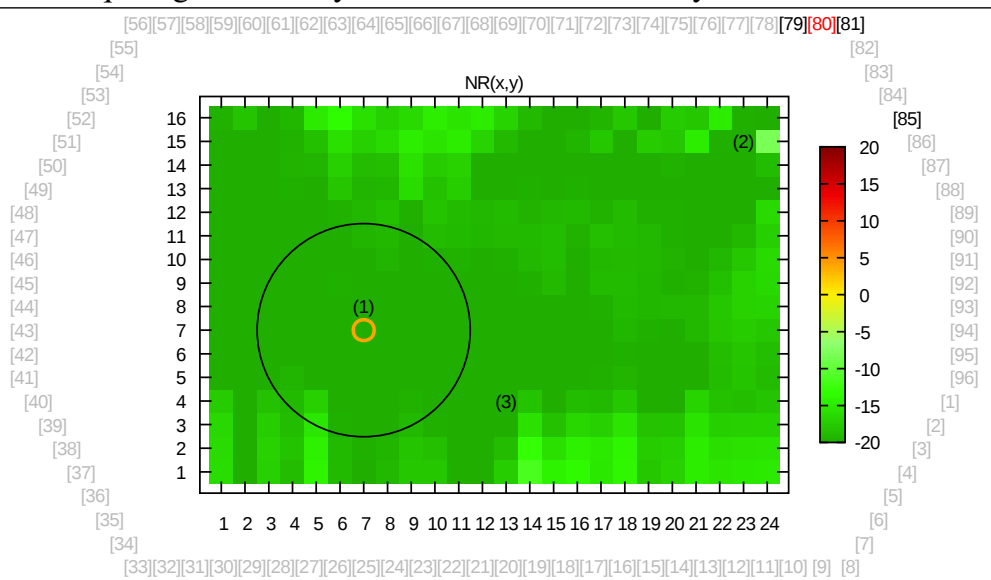

(a) Spatial distributions of the steady-state noise reduction level $\mathrm{NR}(x, y)$ for $M_{s}=1$, given in $\mathrm{dB}$.

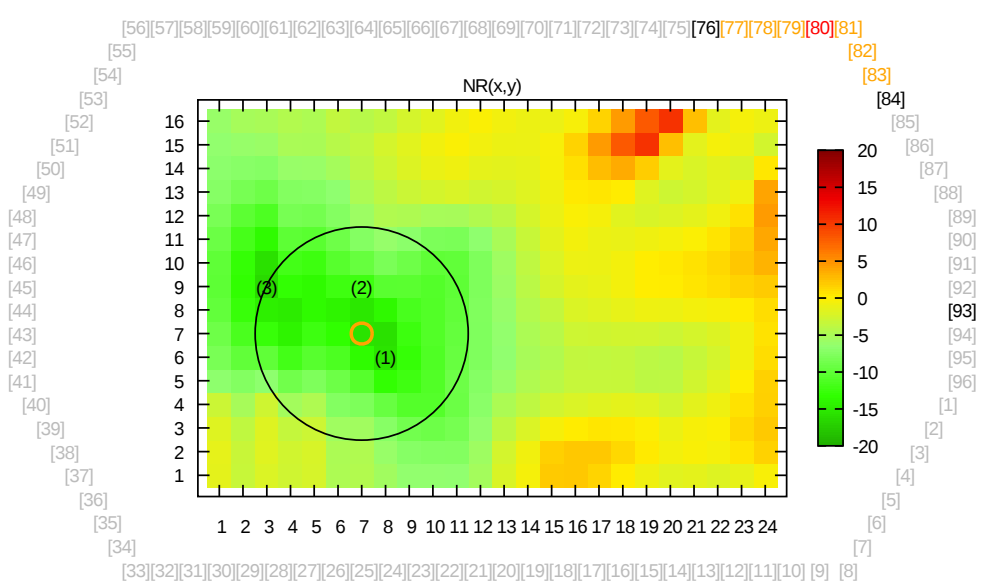

(b) Spatial distributions of the steady-state noise reduction level $\mathrm{NR}(x, y)$ for $M_{s}=4$, given in $\mathrm{dB}$.

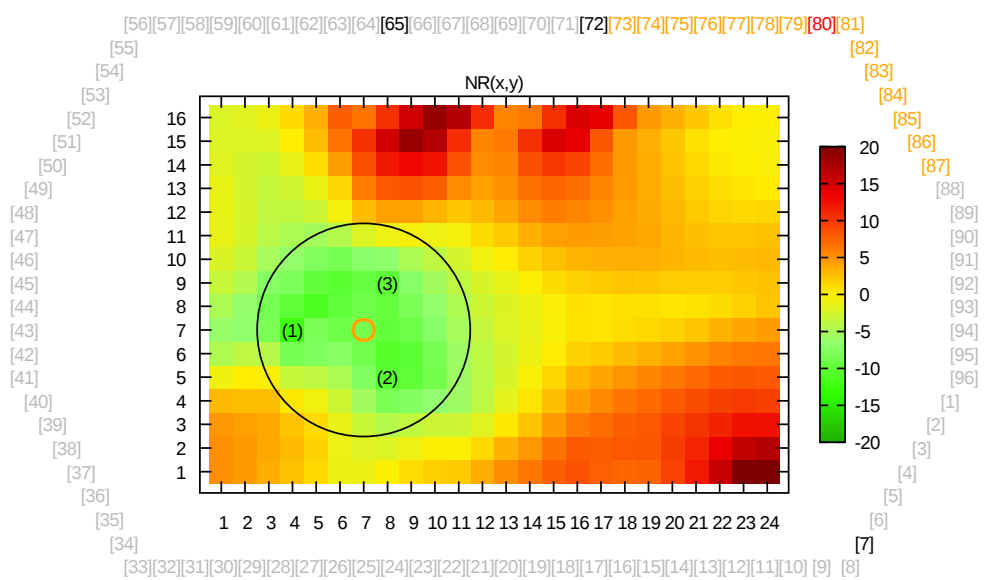

(c) Spatial distributions of the steady-state noise reduction level $\mathrm{NR}(x, y)$ for $M_{s}=8$, given in $\mathrm{dB}$.

Figure 13: Spatial distribution charts of the steady-state noise reduction levels $\mathrm{NR}(x, y)$ for different values of the margin from noise source $M_{s}$. The experiments has been performed for $N_{n}=3, c_{x}=7, c_{y}=7, c_{r}=4$, and $M_{c}=1$. The black and orange circles represents the target circle and the margin from the target circle centre $M_{c}$, respectively. 
- if primary and secondary paths are very similar, noise reduction obtained at a single spot can provide global noise reduction.

When the value of $M_{s}$ increases to 4 (Fig. 13b), a second stage is observed, which constitutes a transient stage to the third one. The effect of global reduction is weakened, but still it is possible to generate a massive zone of quiet and small zones where the noise is enhanced. For the presented study, a threshold is reached approximately at $M_{s}=8$, when the global reduction is lost entirely and the ANC system generates a local zone of quiet (Fig. 13c). Although, it is worth pointing out that the zone of demanded size has been obtained. When the $M_{s}$ increases further, the changes in noise reduction performance are slight and the character on generated zones of quiet is kept the same. 


\subsection{Margin around the zone of quiet centre}

Second evaluation is related to the impact of the margin from the user location, $M_{c}$. The $M_{c}$ parameter takes integer values greater or equal to zero, denoting minimal distance between the user location and the nearest error microphone. A series of evaluations of the shaping method has been performed for different values of $M_{s}$ (in the range from 0 to 8 ). The other parameters took constant values: $c_{x}=7, c_{y}=7, c_{r}=2$, and $M_{s}=10$. The value of $M_{s}$ was arbitrary chosen to represent some obstacles placed around a noise source, what often is encountered in real life. Resulting relation between optimization index $J$, margin $M_{c}$, and number of nodes $N_{n}$ is presented in Fig. 14. In addition, for three selected values of the $M_{c}$ (equal 1, 2 and 4, respectively) spatial distributions of $\mathrm{NR}(x, y)$ are presented in Fig. 15 .

For low values of $M_{c}$, the microphones are concentrated within the target circle and it enables generation of a local zone of quiet with good average mean reduction values. There is almost no difference between $M_{c}=0$ and $M_{c}=1$, which means that error microphones do not have to be present at the exact centre of the target circle to enable good performance. It can be explained by minimal primary disturbance wavelength, which is approximately $1.2 \mathrm{~m}$ in this study. The

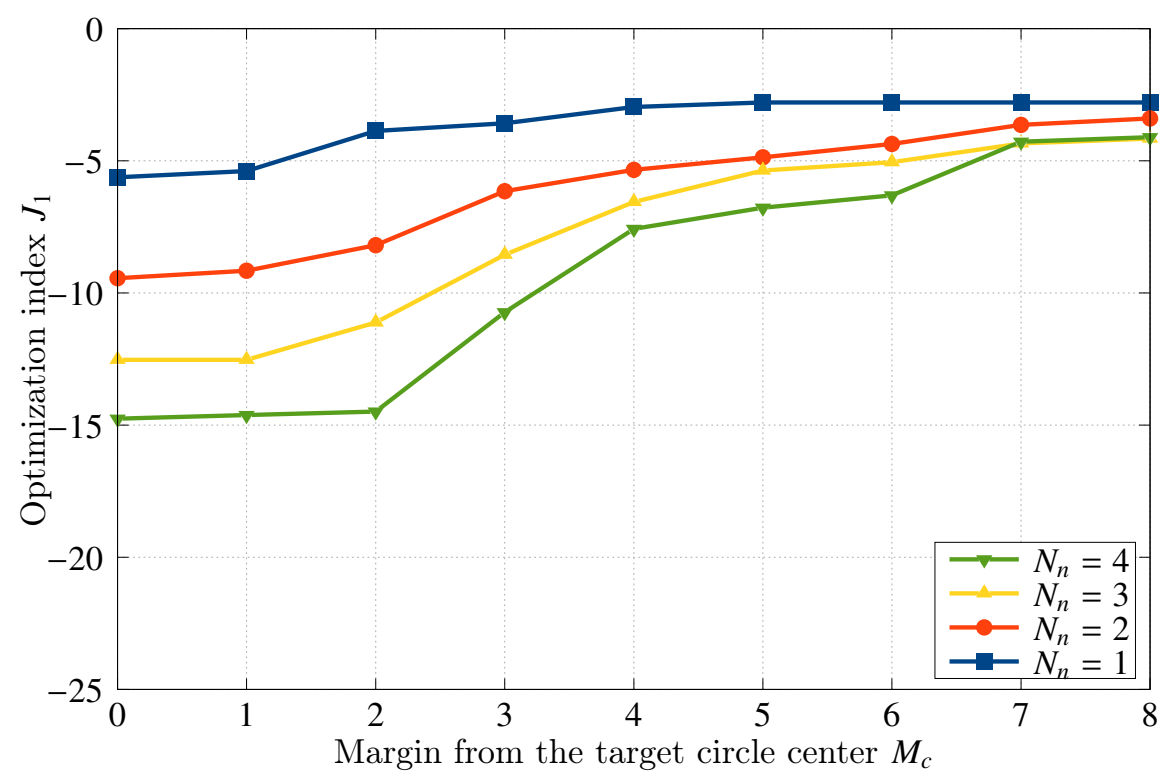

Figure 14: A plot representing relationships between the margin from the target circle centre $M_{c}$, the number of nodes $N_{n}$, and the optimization index $J$. The experiments has been performed for $c_{x}=7, c_{y}=7, c_{r}=2$, and $M_{s}=10$. 
Shaping zones of quiet generated by an active noise control system

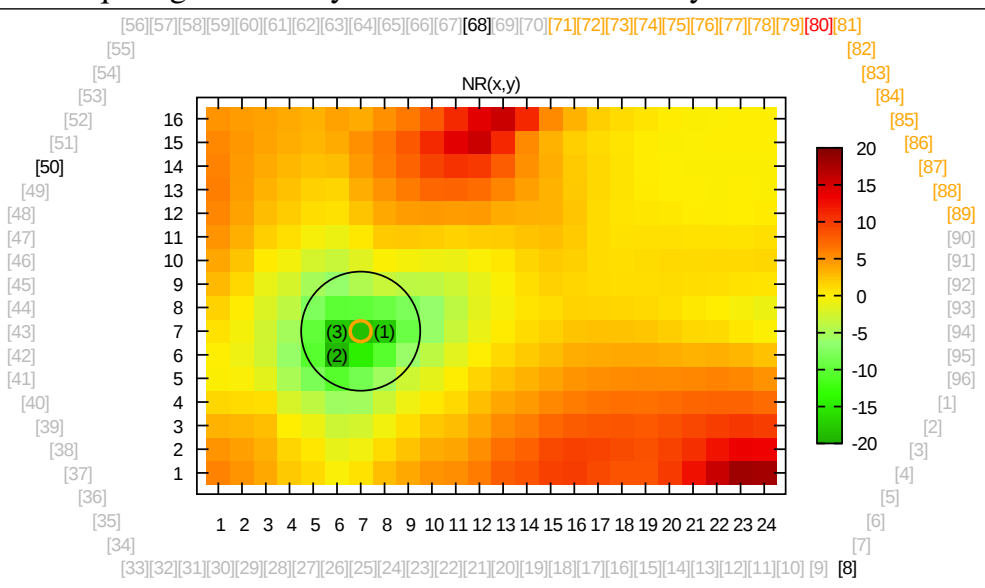

(a) Spatial distributions of the steady-state noise reduction level $\mathrm{NR}(x, y)$ for $M_{c}=1$, given in $\mathrm{dB}$.

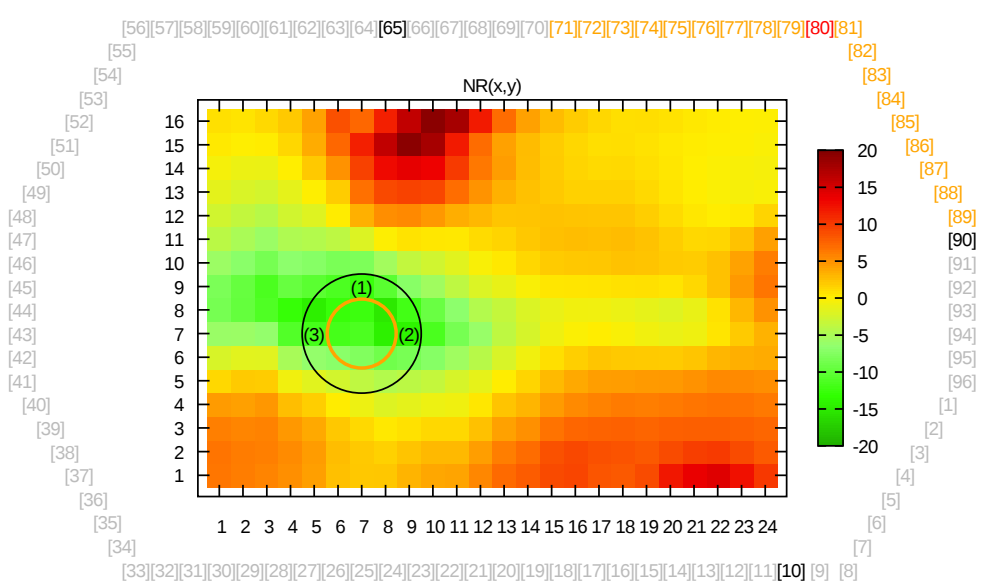

(b) Spatial distributions of the steady-state noise reduction level $\mathrm{NR}(x, y)$ for $M_{c}=2$, given in $\mathrm{dB}$.

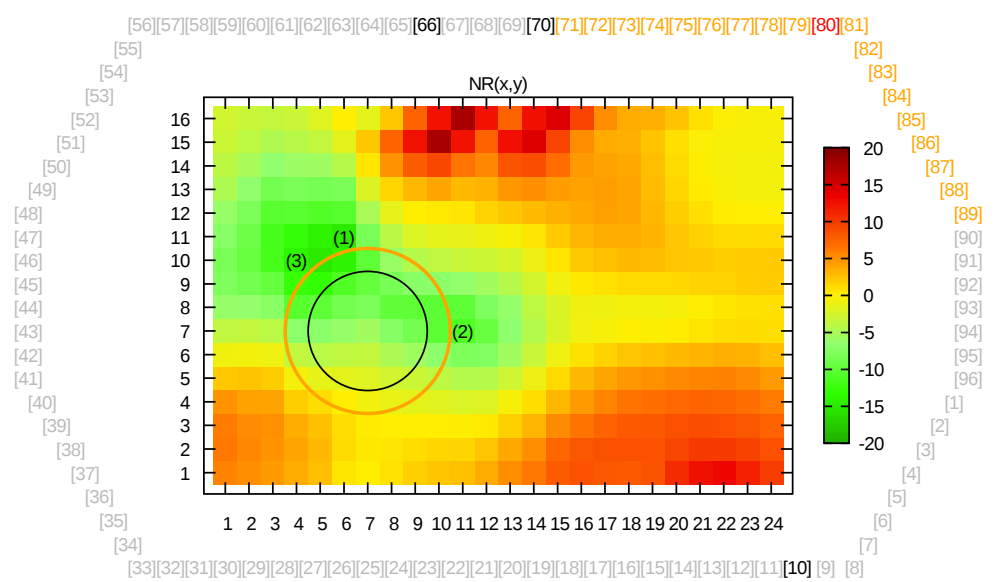

(c) Spatial distributions of the steady-state noise reduction level $\mathrm{NR}(x, y)$ for $M_{c}=4$, given in $\mathrm{dB}$.

Figure 15: Spatial distribution charts of the steady-state noise reduction levels $\mathrm{NR}(x, y)$ for different values of the margin from the target circle centre $M_{c}$. The experiments has been performed for $N_{n}=3, c_{x}=7, c_{y}=7, c_{r}=2$, and $M_{s}=10$. The black and orange circles represents the target circle and the margin from the target circle centre $M_{c}$, respectively. 
Shaping zones of quiet generated by an active noise control system

difference between putting a microphone in one location and any of the neighbouring positions is a distance of $0.2 \mathrm{~m}$, which is less than $1 / 4$ of the minimal primary disturbance wavelength. Therefore, the microphone signal provides information not only about its exact location, but also represent the surrounding positions. When the value of $M_{c}$ increases further, the best possible reduction drops significantly, up to a point where some reduction is provided, but it is rather weak. 


\subsection{Radius of the target circle}

The impact of the radius of the target circle $c_{r}$ is studied in this Subsection. The $c_{r}$ parameter takes integer values greater or equal to zero, denoting the radius of target circle where the zone of quiet is expected to be formed. In other words, it determines on how wide area (around the user) the $\operatorname{NR}(x, y)$ values should be averaged in the optimization index $J$. A final series of evaluations of the shaping method has been performed for different values of the $c_{r}$ (in the range from 0 to 8). The other parameters took constant values: $c_{x}=7, c_{y}=7, M_{c}=1$, and $M_{s}=10$. Resulting relation between optimization index $J$, radius $c_{r}$, and number of nodes $N_{n}$ is presented in Fig. 16 In addition, for three selected values of the $c_{r}$ (equal 1, 3 and 6 , respectively) spatial distributions of $\mathrm{NR}(x, y)$ are presented in Fig. 17

It follows from the analysis of Figs. 16 17 that the wider zone of quiet is expected, the average noise reduction level is lower. It is actually consistent with the intuition. With the same resources, the reduction can be greater at a narrower area, or lesser at a broader area. The characteristics in Fig. 16 shows how fast the mean reduction drops with the increase of radius $c_{r}$. On the other hand, it can be observed how the mean reduction can be increased by adding more control loudspeakers. Knowledge about such trends may be helpful in making a decision, if it is worth to add another control input.

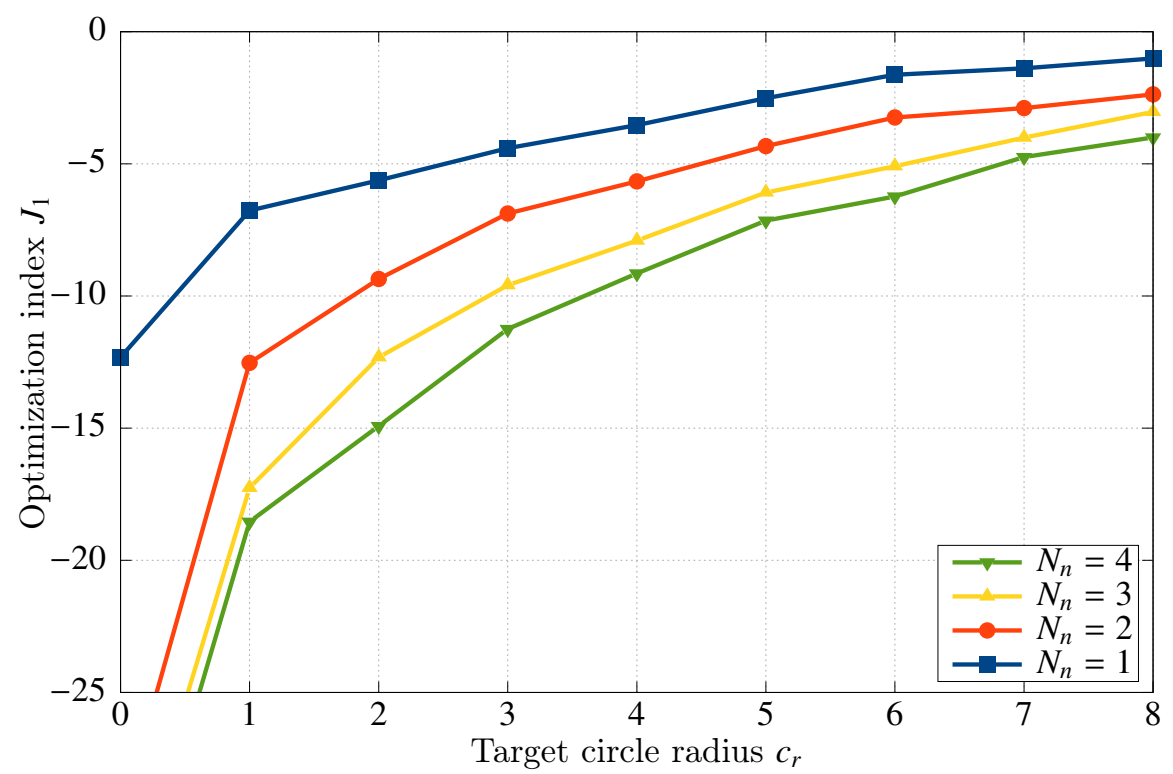

Figure 16: A plot representing relationships between the target circle radius $c_{r}$, the number of nodes $N_{n}$, and the optimization index $J$. The experiments has been performed for $c_{x}=7, c_{y}=7, M_{c}=1$, and $M_{s}=10$. 


\section{Shaping zones of quiet generated by an active noise control system}

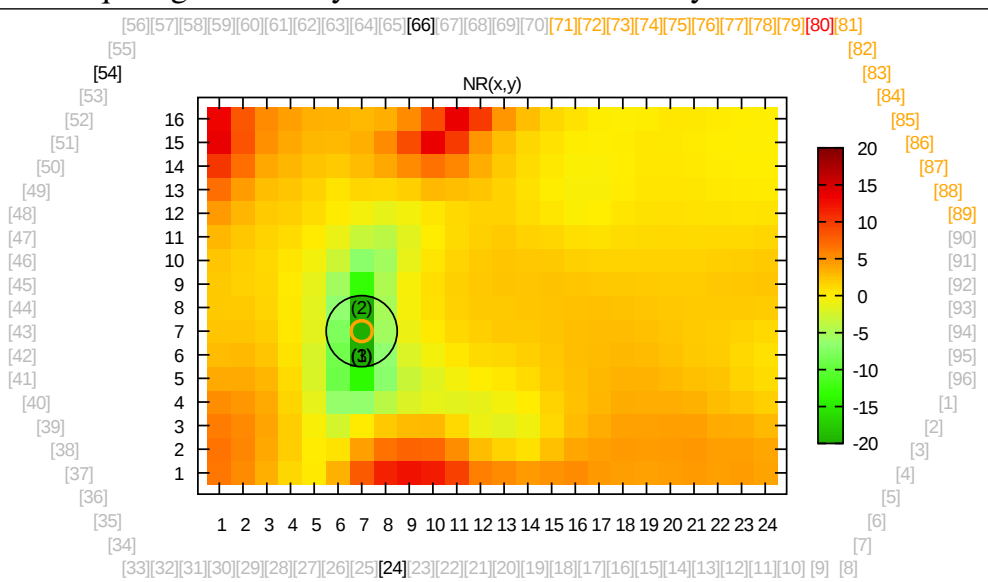

(a) Spatial distributions of the steady-state noise reduction level $\mathrm{NR}(x, y)$ for $c_{r}=1$, given in $\mathrm{dB}$.

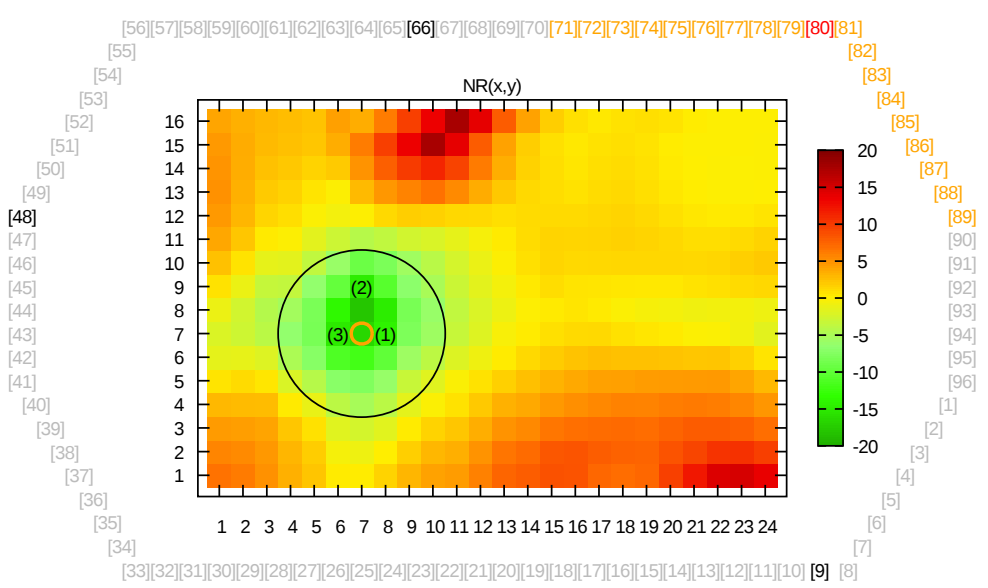

(b) Spatial distributions of the steady-state noise reduction level $\mathrm{NR}(x, y)$ for $c_{r}=3$, given in $\mathrm{dB}$.

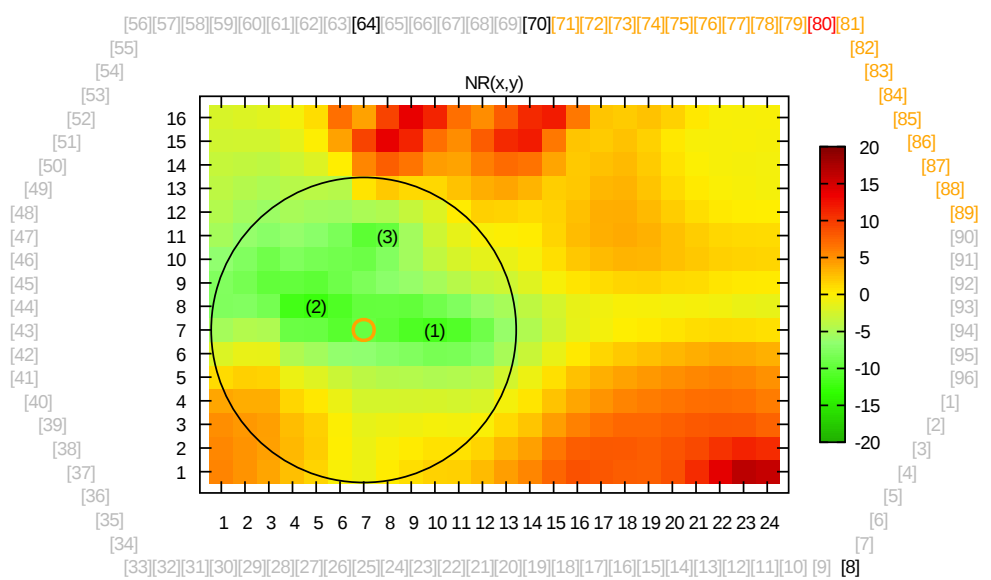

(c) Spatial distributions of the steady-state noise reduction level $\mathrm{NR}(x, y)$ for $c_{r}=6$, given in $\mathrm{dB}$.

Figure 17: Spatial distribution charts of the steady-state noise reduction levels $\mathrm{NR}(x, y)$ for different values of the target circle radius $c_{r}$. The experiments have been performed for $N_{n}=3, c_{x}=7, c_{y}=7, M_{c}=1$, and $M_{s}=10$. The black and orange circles represents the target circle and the margin from the target circle centre $M_{c}$, respectively. 


\subsection{Progress of the optimization}

In this subsection an insight into the working of the optimization algorithm is given. The progress of the optimization carried out using MA is presented in Fig. 18. A comparison of twelve optimization curves performed for the same input data is showed. Additionally, an mean curve is also given.

It follows from the analysis of optimization curves given in Fig. 18 that the MA converges quickly, reaching nearly optimal solutions in less than 12 generations. Even better consistency could be obtained if more generations are allowed or bigger populations would be used, although it increases proportionally the calculation time. To provide a reference, for the given parameters of optimization algorithm, a complete optimization process takes less than an hour on a regular desktop computer. The analysis of computational complexity of the MA depends on a given application - it depends on the used operators. However, the computational demand of the MA has been a subject of investigation in [24, 26].

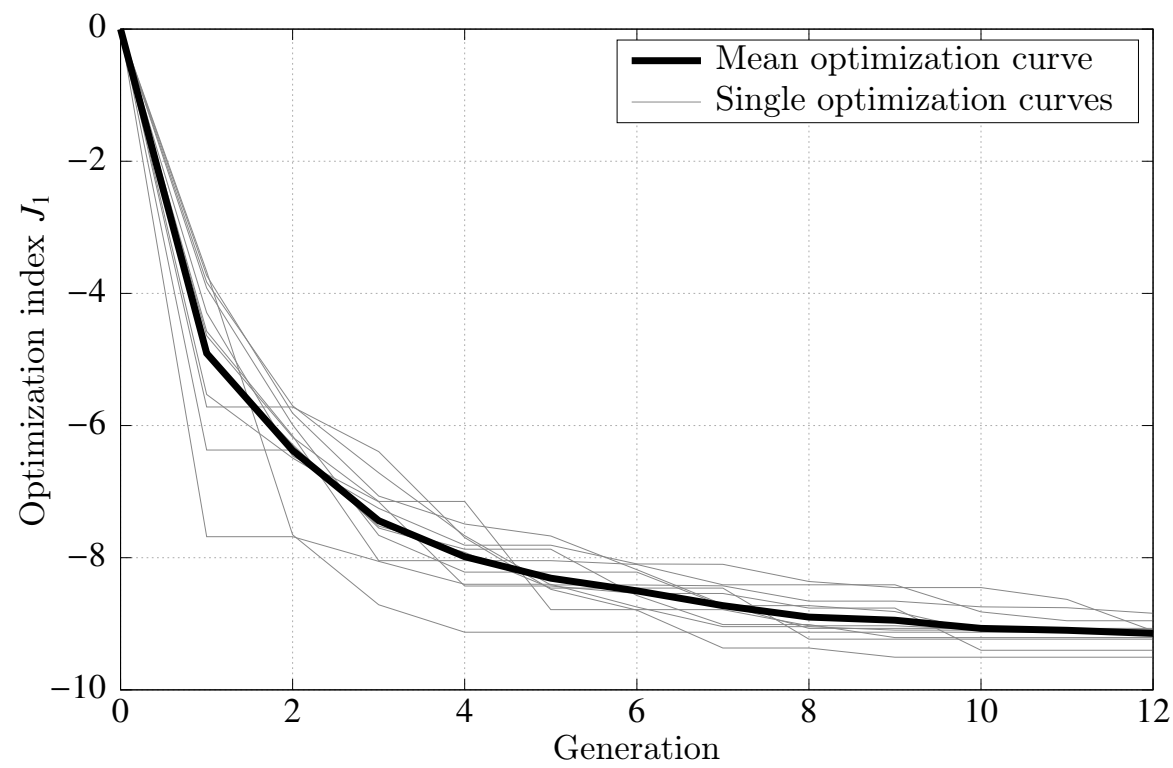

Figure 18: A comparison of twelve optimization curves performed for the same input data. Additionally, an mean curve is also given. The presented optimization has been performed for $N_{n}=3, c_{x}=7, c_{y}=7, c_{r}=3, M_{c}=1$, and $M_{s}=10$. 


\section{Conclusions}

The active noise control techniques are gaining still growing attention in the noise-polluted world. The methods that enhance their performance are therefore important and needed. This paper develops the method for enhancing Noise Reduction (NR) levels and shaping spatial distribution of zones of quiet generated with an ANC system by optimization of sensors and actuators arrangement. To facilitate the explanations, an exemplary scenario is introduced. The method is based on an optimization approach utilizing a computationally-efficient Memetic Algorithm (MA), hence, the problem is appropriately formulated to enable application of the optimization algorithm. The MA operators for the given problem are proposed and described. The precisely designed spatial distribution of target zones is embedded in the cost function (leading to shaped zones of quiet), instead of employing a scalar global performance index. With all its elements defined, the method is ready to be employed in practical applications.

Extensive simulation studies of the proposed method are undertaken. As an example of the acoustic environment, a real large room located at the Audio Processing Laboratory of the Polytechnic University of Valencia has been utilized. It was equipped with 96 loudspeakers generating acoustic excitation and 384 microphones locations acquiring the responses, what resulted in identification of 36864 acoustic responses in total. The influence of several parameters on the obtained noise reduction is evaluated, including size of the demanded zone of quiet, the number of nodes, and constraints imposed on the admissible actuators and sensors locations. Obtained results confirm a high potential of the proposed method.

The method depends on a model of the acoustic environment, which have to be obtained before employing the method. To receive satisfactory results, the model should be sufficiently accurate. However, it can be acquired by any method, experimental or theoretical. The obtained results may also be vulnerable to significant reorganization of the objects present within the

room. In the worst case, the model would have to be updated and the method rerun. However, small changes in the environment should not affect in a significant way the obtained performance, but the vulnerability of the method to the environment changes should be assessed in a separate study. 
Shaping zones of quiet generated by an active noise control system

\section{Acknowledgment}

The authors are indebted to anonymous reviewers for their precious comments and suggestions, which helped improving the paper. The research reported in this paper has been supported by the National Science Centre, Poland, decision no. DEC-2017/25/B/ST7/02236, by the Ministry of Science and Higher Education, Poland, and by EU together with Spanish Government under Grant TEC2015-67387-C4-1-R (MINECO/FEDER).

\section{References}

[1] M. de Diego, A. Gonzalez, M. Ferrer, and G. Piñero. Multichannel active noise control system for local spectrum reshaping of multifrequency noise. Journal of Sound and Vibration, 1-2:249-271, 2004.

[2] C. Antoñanzas, M. Ferrer, M. de Diego, and A. Gonzalez. Blockwise frequency domain active noise controller over distributed networks. Applied Sciences, 6(5):124, 2016.

[3] G. Liu, K. Lu, D. Zou, Z. Xie, Z. Rao, and N. Ta. Development of a semi-active dynamic vibration absorber for longitudinal vibration of propulsion shaft system based on magnetorheological elastomer. Smart Mater. Struct, 26(075009):075009, 2017.

[4] J. Tuma, P. Suranek, M. Mahdal, and R. Wagnerova. Linear piezoactuators in systems of the active vibration control. In Proceedings of the $23^{\text {nd }}$ International Congress of Sound and Vibration, Athens, Greece, pages 10-14, 2016.

[5] A. Preumont. Vibration control of active structures: an introduction, volume 50. Springer Science \& Business Media, 2012

[6] A. Montazeri and J. Poshtan. Ga-based optimization of a mimo anc system considering coupling of secondary sources in a telephone kiosk. Applied Acoustics, 70(7):945-953, 2009.

[7] C. R. Duke, S. D. Sommerfeldt, K. L. Gee, and C. V. Duke. Optimization of control source locations in free-field active noise control using a genetic algorithm. Noise Control Engineering Journal, 57(3):221-231, 2009.

[8] Z. Diamantis, D. Tsahalis, and I. Borchers. Optimization of an active noise control system inside an aircraft, based on the simultaneous optimal positioning of microphones and speakers, with the use of a genetic algorithm. Computational optimization and applications, 23(1):65-76, 2002.

[9] L. Huideng and Q. Arui. Space active noise control system design with multi-objective genetic algorithms. In Evolutionary Computation (CEC), 2011 IEEE Congress on, pages 2186-2192. IEEE, 2011.

[10] K. Baek and S. Elliott. Natural algorithms for choosing source locations in active control systems. Journal of Sound and Vibration, 186(2):245-267, 1995.

[11] D. Manolas, I. Borchers, and D. Tsahalis. Simultaneous optimization of the sensor and actuator positions for an active noise and/or vibration control system using genetic algorithms, applied in a dornier aircraft. Engineering Computations, 17(5):620-630, 2000.

[12] D. Li and M. Hodgson. Optimal active noise control in large rooms using a "locally global" control strategy. The Journal of the Acoustical Society of America, 118(6):3653-3661, 2005. 
Shaping zones of quiet generated by an active noise control system

[13] M. Ferrer, M. de Diego, G. Piñero, and A. Gonzalez. Active noise control over adaptive distributed networks Signal Processing, 107:82-95, 2015.

[14] S. Elliott, I. Stothers, and P. Nelson. A multiple error lms algorithm and its application to the active control of sound and vibration. IEEE Transactions on Acoustics, Speech, and Signal Processing, 35(10):1423-1434, Oct 1987.

[15] S. Wrona and M. Pawelczyk. Active reduction of device multi-tonal noise by controlling vibration of multiple walls of the device casing. In Proceedings of the 19th International Conference on Methods and Models in Automation and Robotics (MMAR), Miedzyzdroje, Poland, pages 687-692, 2014.

[16] S. Wrona and M. Pawelczyk. Feedforward control of a light-weight device casing for active noise reduction. Archives of Acoustics, 41(3):499-505, 2016.

[17] K. Mazur, S. Wrona, and M. Pawelczyk. Design and implementation of multichannel global active structural acoustic control for a device casing. Mechanical Systems and Signal Processing, 98C:877-889, 2018.

[18] J. Wiora, S. Wrona, and M. Pawelczyk. Evaluation of measurement value and uncertainty of sound pressure level difference obtained by active device noise reduction. Measurement, 96:67-75, 2017.

[19] C. G. Lopes and A. H. Sayed. Incremental adaptive strategies over distributed networks. IEEE Transactions on Signal Processing, 55(8):4064-4077, Aug 2007.

[20] D. Bismor, K. Czyz, and Z. Ogonowski. Review and comparison of variable step-size LMS algorithms. International Journal of Acoustics and Vibration, 21(1):24-39, 2016.

[21] C. Antoñanzas, M. Ferrer, M. de Diego, and A. Gonzalez. Control effort strategies for acoustically coupled distributed acoustic nodes. Wireless Communications and Mobile Computing, 2017, 2017.

[22] B. Widrow and S. Stearns. Adaptive signal processing. Englewood Cliffs, NJ, Prentice-Hall, Inc, 1985.

[23] D. E. Goldberg and J. H. Holland. Genetic algorithms and machine learning. Machine learning, 3(2):95-99, 1989.

[24] F. Neri, C. Cotta, and P. Moscato. Handbook of memetic algorithms, volume 379. Springer, 2012.

[25] S. Wrona and M. Pawelczyk. Optimal placement of actuators for active structural acoustic control of a light-weight device casing. In Proceedings of 23rd International Congress on Sound and Vibration, Athens, Greece, 10-14 July, 2016.

[26] P. Garg. A comparison between memetic algorithm and genetic algorithm for the cryptanalysis of simplified data encryption standard algorithm. International Journal of Network Security and Its Applications, 1(1):34-42, 2009. 
Shaping zones of quiet generated by an active noise control system

\section{Nomenclature}

\begin{tabular}{|c|c|}
\hline $\mathrm{A}_{\mathrm{i}}(\mathbf{x})$ & $i$ th shape function at position $\mathbf{x}$ \\
\hline$c_{x}, c_{y}$ & coordinates of the target circle centre \\
\hline$c_{r}$ & radius of the target circle \\
\hline$d(\mathbf{x}, n)$ & primary disturbance signal at position $\mathbf{x}$ and time $n$ \\
\hline$D_{a}$ & numbers of dimensions of space where actuators locations are defined \\
\hline$D_{s}$ & numbers of dimensions of space where sensors locations are defined \\
\hline$e_{k}(n)$ & error signal obtained at $k$ th node \\
\hline$e(\mathbf{x}, n)$ & error signal at position $\mathbf{x}$ and time $n$ \\
\hline$i, j, k$ & positive integers \\
\hline$J_{i}$ & $i$ th cost function \\
\hline$L_{s}$ & order of the FIR models of secondary paths \\
\hline$L_{w}$ & control filter order \\
\hline$M_{c}$ & margin from the user location \\
\hline$M_{s}$ & margin from the noise source \\
\hline$n$ & time, defined in integer sample numbers \\
\hline$N_{a}$ & total number of actuators \\
\hline$N_{c}$ & total number of coordinates describing arrangement of sensors and actuators \\
\hline$N_{n}$ & number of ANC system nodes \\
\hline$N_{s}$ & total number of sensors \\
\hline$N_{\exp }$ & number of samples generated in an experiment \\
\hline $\mathrm{NR}(\mathbf{x})$ & steady-state noise reduction level at point $\mathbf{x}$ \\
\hline $\mathrm{NR}(\mathbf{x}, n)$ & instantaneous noise reduction level \\
\hline$p$ & real number denoting the fraction of the experiment when a steady-state is reached \\
\hline $\mathbf{r}_{k}(n)$ & $k$ th filtered-reference concatenated vector \\
\hline $\mathbf{r}_{i k}(n)$ & vector of regressors of the $i k$ th filtered-reference signal \\
\hline$r_{i k}(n)$ & $i k$ th filtered-reference signal \\
\hline$\hat{\mathbf{s}}_{i k}$ & vector of coefficients of the FIR model of the $i k$ th secondary path \\
\hline$u_{k}(n)$ & $k$ th control signal \\
\hline $\mathbf{w}(n)$ & global control filter vector \\
\hline $\mathbf{w}_{k}(n)$ & $k$ th control filter used at $k$ th node \\
\hline
\end{tabular}


Shaping zones of quiet generated by an active noise control system

$\begin{array}{ll}x(n) & \text { reference signal } \\ \mathbf{x}_{r}(n) & \text { vector of regressors of the reference signal (of length equal to } L_{s} \text { ) } \\ \mathbf{x}_{u}(n) & \text { vector of regressors of the reference signal (of length equal to } L_{w} \text { ) } \\ \mathbf{x} & \text { coordinates describing a point in the observation zone } \\ \mathbf{x}_{a, i} & \text { coordinates describing location of the } i \text { th actuator } \\ \mathbf{x}_{s, i} & \text { coordinates describing location of the } i \text { th sensor } \\ x_{a, i, j} & j \text { th coordinate of } i \text { th actuator } \\ x_{s, i, j} & j \text { th coordinate of } i \text { th sensor } \\ \alpha & \text { leakage coefficient } \\ \mu & \text { step-size parameter } \\ (x, y) & \text { coordinates describing a point in the observation zone }\end{array}$

